\title{
LOCAL VOLATILITY DYNAMIC MODELS
}

\author{
RENÉ CARMONA AND SERGEY NADTOCHIY \\ BENDHEIM CENTER FOR FINANCE, ORFE \\ PRINCETON UNIVERSITY \\ PRINCETON, NJ 08544 \\ RCARMONA@PRINCETON.EDU \& SNADTOCH@PRINCETON.EDU
}

\begin{abstract}
This paper is concerned with the characterization of arbitrage free dynamic stochastic models for the equity markets when Itô stochastic differential equations are used to model the dynamics of a set of basic instruments including, but not limited to, the underliers. We study these market models in the framework of the HJM philosophy originally articulated for Treasury bond markets. The approach to dynamic equity models which we follow was originally advocated by Derman and Kani in a rather informal way. The present paper can be viewed as a rigorous development of this program, with explicit formulae, rigorous proofs and numerical examples.
\end{abstract}

Keywords Implied volatilty surface - Local Volatility surface - Market models - Arbitrage-free term structure dynamics - HeathJarrowMorton theory.

Mathematics Subject Classification (2000) 91B24

JEL Classification (2000) G13

\section{INTRODUCTION AND NOTATION}

Most financial market models introduced for the purpose of pricing and hedging derivatives concentrate on the dynamics of the underlying stocks, or underlying instruments on which the derivatives are written. This is clearly the case in the Black-Scholes theory where the focus is on the dynamics of the underlying stocks, whether they are assumed to be given by geometric Brownian motions or more general non-negative diffusions, or even semi-martingales with jumps. In contrast, the focus of the present paper is on the simultaneous dynamics of all the liquidly traded derivative instruments written on the underlying stocks.

For the sake of simplicity, we limit ourselves to a single underlying index or stock on which all the derivatives under consideration are written. Choosing more underliers would force the price process to be multivariate and make the notation significantly more complicated, unnecessarily obscuring the nature of the results.

We denote by $\left\{S_{t}\right\}_{t \geq 0}$ the price process underlying the derivative instruments forming the market. In order to further simplify the notation, we assume that the discount factor is one, or equivalently

\footnotetext{
${ }^{1}$ The results of this paper were presented, starting September 2006, in a number of seminars including Columbia, Cornell, Kyoto, Santa Barbara, Stanford, Banff, Oxford, etc. and the organizers and participants are thanked for numerous encouraging discussions.

${ }^{2}$ Corresponding author: René Carmona

Date: May28, 2007.
} 
that the short interest rate is zero, i.e. $r_{t} \equiv 0$, and that the underlying stock does not pay dividends. These assumptions greatly simplify the notation without affecting the generality of our derivations.

We assume that in our idealized market, European call options of all strikes and maturities are traded, that their prices are observable, and that they can be bought or sold at these prices in any quantity. We denote by $C_{t}(T, K)$ the market price at time $t$ of a European call option of strike $K$ and maturity $T>t$. We assume that today, i.e. on day $t=0$, all the prices $C_{0}(T, K)$ are observable. According to the philosophy of market models adopted in this paper, at any given time $t$, instead of working directly with the price $S_{t}$ of the underlying asset, we use the set of call prices $\left\{C_{t}(T, K)\right\}_{T \geq t, K \geq 0}$ as our fundamental market data. This is partly justified by the well documented fact that many observed option price movements cannot be attributed to changes in $S_{t}$, and partly by the fact that many exotic (path dependent) options are hedged (replicated) with portfolios of plain (vanilla) call options.

Note that in our idealized market, we assume that European call options of all strikes and all maturities are liquidly traded. This assumption is highly unrealistic. In practice, the best one can hope for is, for a finite set of discrete maturities $T_{1}<T_{2}<\cdots<T_{n}$, quotes for the prices $C_{t}\left(T_{i}, K_{i j}\right)$ of a finite set of call options. In other words, for each of the maturities $T_{i}$, prices of calls are only available for a finite set $K_{i 1}<K_{i 2}<\cdots<K_{i n_{i}}$ of strikes. This more realistic form of the set-up has been considered, starting with the work of Laurent and Leisen [24], followed by the recent technical reports by Cousot [9] and Buehler [5] who use Kellerer [22] theorem, and by the recent work of Davis and Hobson [10] which relies instead on the Sherman-Stein-Blackwell theorem [35, 36, 2].

For the sake of convenience, we denote by $\tau=T-t$ the time to maturity of the option and we denote by $\tilde{C}_{t}(\tau, K)$ the price $C_{t}(T, K)$ expressed as a function of this new variable. In other words

$$
\tilde{C}_{t}(\tau, K)=C_{t}(t+\tau, K), \quad \tau>0, K>0 .
$$

Despite the shortcomings which we will have to deal with, this switch in notation has the clear advantage of casting the data at each time $t$, into a surface parameterized over a range $\{(\tau, K) ; \tau>$ $0, K>0\}$ which does not change with $t$. We work in a linear pricing system. In other words, we assume that the market prices by expectation in the sense that the prices of the liquid instruments are given by expectations of the present values of their cashflows. So saying that $\mathbb{Q}$ is a pricing measure used by the market implies that for each time $t \geq 0$ we have

$$
\tilde{C}_{t}(\tau, K)=\mathbb{E}\left\{\left(S_{t+\tau}-K\right)^{+} \mid \mathcal{F}_{t}\right\}=\mathbb{E}^{\mathbb{Q}_{t}}\left\{\left(S_{t+\tau}-K\right)^{+}\right\} .
$$

if we denote by $\mathbb{Q}_{t}$ a regular version of the conditional probability of $\mathbb{Q}$ with respect to $\mathcal{F}_{t}$. For each $\tau>0$, we denote by $\tilde{\mu}_{t, t+\tau}$ the distribution of $S_{t+\tau}$ for the conditional distribution $\mathbb{Q}_{t}$. It is an $\mathcal{F}_{t^{-}}$ measurable random measure. With this notation

$$
\tilde{C}_{t}(\tau, K)=\int_{0}^{\infty}(x-K)^{+} d \tilde{\mu}_{t, t+\tau}(d x)
$$

and for each fixed $\tau>0$, the knowledge of the prices $\tilde{C}_{t}(\tau, K)$ for all the stikes $K>0$ completely determines the distribution $\tilde{\mu}_{t, t+\tau}$ on $[0, \infty)$.

Notice that we do not assume uniqueness of the pricing measure $\mathbb{Q}$. In other words, our analysis holds in the case of incomplete models as well as complete models. 
Notation Convention. In order to help with the readability of the paper, we use a notation without a tilde or a hat for all the quantities expressed in terms of the variables $T$ and $K$. But we shall add a tilde when such a quantity is expressed in terms of the variables $\tau$ and $K$, and a hat when the strike is given in terms of the variable $x=\log K$.

Implied Volatility Code-Book In the classical Black-Scholes theory, the dynamics of the underlying asset are given by the stochastic differential equation

$$
d S_{t}=S_{t} \sigma d W_{t}, \quad S_{0}=s_{0}
$$

for some univariate Wiener process $\left\{W_{t}\right\}_{t}$ and some positive constant $\sigma$. In this case, the price $\tilde{C}_{t}(\tau, K)$ of a call option is given by the Black-Scholes formula

$$
B S(S, \tau, \sigma, K)=S_{t} \Phi\left(d_{1}\right)-K \Phi\left(d_{2}\right)
$$

with

$$
d_{1}=\frac{-\log M_{t}+\tau \sigma^{2} / 2}{\sigma \sqrt{\tau}}, \quad d_{2}=\frac{-\log M_{t}-\tau \sigma^{2} / 2}{\sigma \sqrt{\tau}}
$$

where $M_{t}=K / S_{t}$ is the moneyness of the option and where we use the notation $\Phi$ for the cumulative distribution of the standard normal distribution. The Black-Scholes price is an increasing function of the parameter $\sigma$ when all the other parameters are held fixed. As a consequence, for every real number $C$ (think of such a number as a quoted price for a call option with time to maturity $\tau$ and strike $K$ ) in the interval between $\left(S_{t}-K\right)^{+}$and $S_{t}$, there exists a unique number $\sigma$ for which $\tilde{C}_{t}(\tau, K)=C$. This unique value of $\sigma$ given by inverting Black-Scholes formula (2) is known as the implied volatility and we shall denote it by $\tilde{\Sigma}_{t}(\tau, K)$. This quantity is extremely important as it is used by most if not all market participants as the currency in which the option prices are quoted. For each time $t>0$, the one-to-one correspondence

$$
\left\{\tilde{C}_{t}(\tau, K) ; \tau>0, K>0\right\} \leftrightarrow\left\{\tilde{\Sigma}_{t}(\tau, K) ; \tau>0, K>0\right\}
$$

offers a code-book translating without loss all the information given by the call prices in terms of implied volatilities. We call it the implied volatility code-book. The mathematical analysis of this surface is based on a subtle mixture of empirical facts and arbitrage theories, and it is rather technical in nature. The literature on the subject is vast and it cannot be done justice in a few references. Choosing a few samples for their relevance to the present discussion, we invite the interested reader to consult [7],[15],[25], [26],[16] and the references therein to get a better sense of these technicalities.

Valuation and risk management of complex option positions require models for the time evolution of implied volatility surfaces. [30] and [8] are early examples of attempts to go beyond static models, but despite the fact that they consider only a cross section of the surface (say for $K$ fixed), the works of Schönbucher [32] and Schweizer and Wissel [34] are more in the spirit of the market model approach which we advocate in this paper.

At any given time $t$, absence of (static) arbitrage imposes conditions on the surface of call option prices: the surface $\left\{\tilde{C}_{t}(\tau, K)\right\}_{\tau, K}$ should be increasing in $\tau$, non-increasing and convex in $K$, it should converge to 0 as $K \rightarrow \infty$ and recover the underlying price $S_{0}$ for zero strike when $K \rightarrow 0$. Because of the one-to-one correspondence between call prices and implied volatilities, these conditions can be expressed in terms of properties of the implied volatility surface $\left\{\tilde{\Sigma}_{t}(\tau, K)\right\}_{\tau, K}$. However inverting Black-Scholes formula (2) is not simple and these conditions become rather technical. 
Moreover, when defining a market model by a set of stochastic differential equations, the first point of the check list is to make sure that these properties guaranteeing the absence of static arbitrage are preserved throughout the time evolution. So even before we consider the more complicated problem of absence of dynamic arbitrage, this first point on the check list gives us enough reasons to search for another way to capture the information contained in the surface of call option prices.

Throughout this paper we assume that the filtration $\mathbb{F}=\mathbb{F}^{W}$ is generated by a multidimensional Brownian motion $\mathbb{W}=\left(W^{1}, \ldots, W^{m}\right)$. We assume that pricing is done by expectation with respect to some pricing measure $\mathbb{Q}$, which, however, doesn't have to be unique. Since it is tradable, the underlying $S_{t}$ must be a local martingale, and the martingale representation theorem for Brownian filtrations gives the existence of an adapted scalar process $\left\{\sigma_{t}\right\}_{t \geq 0}$ and of an $m$-dimensional Wiener process $\mathbb{B}=\left(B^{1}, \ldots, B^{m}\right)$ such that $\mathbb{F}^{W}=\mathbb{F}^{B}$ and

$$
d S_{t}=S_{t} \sigma_{t} d B_{t}^{1}, \quad S_{0}=s_{0} .
$$

In the absence of restrictive assumptions on the particular form of the spot volatility $\sigma_{t}$, pricing by computing expectations of discounted payoffs is usually impractical. An alternative in the spirit of the market models of the fixed income markets would be to postulate the dynamics of the set of option prices explicitly, for example using a system of Itô's stochastic differential equations. However, doing so is likely to introduce arbitrage opportunities and identifying these arbitrage would require much more than a mere modicum of care. As explained earlier, in order to rule out static arbitrages, at any given time, the solution of the system of stochastic differential equations should give a surface increasing in the variable $T$, convex and decreasing in the variable $K$ and satisfying specific boundary conditions. It seems to be quite difficult to identify stochastic dynamics preserving these properties, let alone ruling out dynamic arbitrages! A model based on the simultaneous dynamics of all the call option prices was considered in a recent paper [21] of Jacod and Protter. These authors work in the more general setting of jump processes, and they study the problem of the completion of a market by added derivative instruments. In so doing, they derive conditions very similar to our spot consistency and drift conditions. See also [34] for a discussion of some of the difficulties associated with the simultaneous dynamics of all the call prices.

Equivalently, one could encode all the option prices by the implied volatility surface $\Sigma_{t}$ whose definition was recalled earlier, and model its dynamics. But again, isolating tractable conditions characterizing the absence of arbitrage is very technical and cumbersome. See for example [32] and [34] for a discussion of the particular case when $K$ is fixed.

For all these reasons, we choose to encode the market information (i.e. the option prices) with the so-called local volatility surface. For each fixed time $t \geq 0$, if we assume that $\tilde{C}_{t}(\tau, K)$ is differentiable in $\tau$ and twice differentiable in $K$, we define the local volatility $a_{t}(\tau, K)$ by

$$
\tilde{a}_{t}^{2}(\tau, K)=\frac{2 \partial_{\tau} \tilde{C}(\tau, K)}{K^{2} \partial_{K K}^{2} \tilde{C}(\tau, K)}, \quad \tau>0, K>0 .
$$

In this form, the definition was first given in 1994 by Dupire [14] who considered the case $t=$ 0 , assumed that the underlying was Markovian, and used the theory of the backward Kolmogorov equation to show that the underlier necessarily satisfies a stochastic differential equation of the form

$$
\frac{d S_{t}}{S_{t}}=a\left(t, S_{t}\right) d B_{t}
$$


Dupire's idea was to deduce the dynamics of the underlier from a snapshot at time $t=0$ of the option prices quoted on the market, and use these dynamics to price exotic derivatives on the same underlying. This very same idea appeared approximately at the same time in a work of Derman and Kani in the context of tree models [11]. These groundbreaking works had a tremendous impact on the life on equity desks where pricing and hedging of exotic options take place, and on the research on the mathematical foundations of hedging and pricing of these options. However, this approach is not without shortcomings. First the Markovian property of the underlier is highly unrealistic. Also, the local volatility surface is changing with time, so in general it should be viewed as a function-valued random process $\left\{\tilde{a}_{t}\right\}_{t}$. In some sense, this is the starting point of the present paper.

If we turn definition (3) around, we see that any local volatility surface $\{\tilde{a}(\tau, K)\}_{\tau, K}$ satisfying some mild assumptions (say positivity and continuity for example) gives a set of option prices as a solution of the following initial value problem.

$$
\left\{\begin{array}{l}
\partial_{\tau} \tilde{C}(\tau, K)=\frac{1}{2} K^{2} \tilde{a}^{2}(\tau, K) \partial_{K K}^{2} \tilde{C}(\tau, K), \quad \tau>0, K>0 \\
\tilde{C}_{t}(0, K)=\left(S_{t}-K\right)^{+}
\end{array}\right.
$$

It is important to stress the fact that the four properties which we articulated for the absence of static arbitrage are, in the local volatility framework, encapsulated in the non-negativity of the diffusion coefficient $(\tau, K) \hookrightarrow \tilde{a}_{t}^{2}(\tau, K)$.

As we already mentioned, Schönbucher and Schweizer and Wissel have argued that the implied volatility was not the right code-book for equity market models (see [32] and [34]), and in the simpler case of a cross section they work instead with the term structure of volatility for a fixed option. Our point of view is to follow the spirit of the approach advocated by Schönbucher in the case of credit portfolios [33]. In this approach, using the Breeden-Litzenberger trick, the market prices are put in correspondence with a set of marginal distributions, which is in turn captured by a Markov-martingale having these distributions as marginals. Defining a dynamic model is then done by prescribing a time evolution for this Markov martingale. In the case of the CDO markets, this Markov martingale is a finite state non-homogeneous Markov process with values in the set of possible levels of loss, while here it is a non-homogeneous diffusion in the strike variable. Note that the classical HJM model is included in this approach, the state space of the Markov martingale being a singleton !!! The reader interested in more information on this approach is referred to the survey article [6].

It seems that the terminology local volatility is due to Derman and Kani who studied it in [12] in a paper mostly known for its analysis of tree models. In this work, the authors discuss informally arbitrage-free dynamic models for the local volatility, exactly in the same spirit as the present paper. However, here we develop the program they outline by providing rigorous proofs, new formulae and numerical examples. Also, we obtain different no-arbitrage conditions.

We conclude this introduction with a short summary of the contents of the paper.

In Section 2 we define the time evolution of the (stochastic) volatility surface $a_{t}(\cdot, \cdot)$ by means of a family of Itô's stochastic differential equations. Recall that for each time $t$, the data encapsulated in the surface $a_{t}(\cdot, \cdot)$ give the surface of option prices $C_{t}(\cdot, \cdot)$ by solving the boundary problem (4). Section 3 addresses the obvious issue of the probabilistic characterization of these solutions. Naturally, the individual call prices $C_{t}(T, K)$ are expected to be semi-martinales in $t$. Strangely enough, we could not find a simple proof of this plain fact. Our proof is rather lengthy and technical, and for this reason, the details are postponed to a couple of appendices at the end of the paper. 
The dynamics of the local volatility imply specific dynamics for the set of option prices. One of the thrusts of the paper is to derive necessary and sufficient conditions which guarantee that these dynamics do not produce arbitrage opportunities. We derive such conditions in Section 4. These conditions turn out to be analogous to the drift restriction and short rate specification of the classical Heath-Jarrow-Morton theory (see [6] and [11] for an analog in a similar setup). The following Section 5 characterizes the Markov spot models as those with local volatility bounded variations dynamics. Next, Section 6 provides a new expression for the local volatility surfaces of stochastic volatility models. This expression is especially well suited to Monte Carlo computations. We use intuition based on formulae derived in Section 6 to define a parametric family of local volatility surfaces in Section 7. Even though we believe that, like in the case of the classical HJM models for the bond markets, generic parametric families of local volatility surfaces will not be consistent with the restrictions of the no-arbitrage dynamics (see for example [18] for the classical case), we illustrate the potential usefulness of such parametric families in the analysis of real data. Finally, Section 8 considers the problem of hedging in the framework of the dynamic local volatility stochastic models considered in the paper.

Most of the results of the paper were announced in [6] where the interested reader will find a non-technical introduction to dynamic market models in the form of a survey.

\section{Model Setup}

In this section we mostly work with the variables $\tau$ and $x=\log K$. With this choice, the partial differential equation (4) becomes uniformly parabolic

$$
\partial_{\tau} u(\tau, x)=\frac{1}{2} \tilde{a}_{t}^{2}\left(\tau, e^{x}\right)\left(\partial_{x x}^{2} u(\tau, x)-\partial_{x} u(\tau, x)\right) .
$$

We know that this initial value problem is well-posed and has a classical fundamental solution. We perform the same change of variables in the option prices by defining the function $(\tau, x) \hookrightarrow \hat{C}(\tau, x)$ by:

$$
\hat{C}(\tau, x)=\tilde{C}\left(\tau, e^{x}\right) \quad \text { and } \quad \hat{a}(\tau, x)=\tilde{a}\left(\tau, e^{x}\right) \quad \tau \geq 0, x \in \mathbb{R} .
$$

With this notation, the equation for option prices becomes

$$
\left\{\begin{array}{l}
\partial_{\tau} \hat{C}_{t}(\tau, x)=\frac{1}{2} \hat{a}_{t}^{2}(\tau, x)\left(\partial_{x x}^{2} \hat{C}_{t}(\tau, x)-\partial_{x} \hat{C}_{t}(\tau, x)\right), \tau>0, x \in \mathbb{R} \\
\hat{C}_{t}(0, x)=\left(S_{t}-e^{x}\right)^{+}
\end{array}\right.
$$

We are now ready to postulate the market dynamics. Under $\mathbb{Q}$ our model is given by the following system of stochastic differential equations

$$
\left\{\begin{array}{cc}
d S_{t}=S_{t} \sigma_{t} d B_{t}^{1}, & S_{0} \\
d \hat{a}_{t}^{2}(\tau, x)=\hat{a}_{t}^{2}(\tau, x)\left[\hat{\alpha}_{t}(\tau, x) d t+\hat{\beta}_{t}(\tau, x) \cdot d B_{t},\right] & \hat{a}_{0}^{2}(\tau, x)
\end{array}\right.
$$

where, for each fixed $\tau>0$ and $x \in \mathbb{R}$, the processes $\hat{\alpha}(\tau, x)=\left\{\hat{\alpha}_{t}(\tau, x)\right\}_{t}$ and $\hat{\beta}(\tau, x)=$ $\left\{\hat{\beta}_{t}(\tau, x)\right\}_{t}$ as well as the process $\sigma=\left\{\sigma_{t}\right\}_{t}$ are adapted to the filtration $\mathbb{F}=\mathbb{F}^{B}$. In addition, we will require that the following regularity assumptions are satisfied:

(1) For any $t>0$ it holds that $\mathbb{E} \int_{0}^{t} S_{u}^{2} \sigma_{u}^{2} d u<\infty$.

(2) For each fixed $\tau>0$ and $x \in \mathbb{R}$, $\alpha$ and $\beta$ have continuous derivatives of order one in $\tau$, of order five in $x$, which are adapted continuous processes in $t$. 
(3) Almost surely for any $t \geq 0$ and $T>0$ there exists $\lambda_{*}>0$, such that

$$
\inf _{\tau \in(0, T], x \in \mathbb{R}, u \in[0, t]} \hat{a}_{u}^{2}(\tau, x) \geq \lambda_{*}
$$

and

$$
\sup _{\tau \in(0, T], x \in \mathbb{R}}\left|\partial_{\tau} \hat{a}_{t}^{2}(\tau, x)\right|<\infty .
$$

(4) There exist $\lambda_{1}(t)$ and $\lambda_{2}(t)$, nonnegative adapted processes, with $\mathbb{E} \int_{0}^{t} \lambda_{2}^{5}(s) e^{c \lambda_{1}(s)} d s<\infty$ for any $c \in \mathbb{R}$. Such that, almost surely for all $t \geq 0, \tau>0$ and $x \in \mathbb{R}$

$$
\begin{array}{ll}
\left|\partial_{x^{k}}^{k} \hat{a}_{t}^{2}(\tau, x)\right| \leq \lambda_{1}(t) & k=1,2 \\
\left|\partial_{x^{k}}^{k} \hat{a}_{t}^{2}(\tau, x)\right| \leq \lambda_{2}(t) & k=0,3,4,5 .
\end{array}
$$

(5) Almost surely for all $\tau>0$ and $x \in \mathbb{R}$

$$
\left|e^{-|x|} \partial_{x^{k}}^{k} \hat{\beta}_{t}(\tau, x)\right|+\left|e^{-|x|} \hat{\alpha}_{t}(\tau, x)\right| \leq \lambda_{2}(t), \quad k=0,1,2 .
$$

The first assumption guarantees that $S$ is a martingale. It will play an important role in some of the proofs. The second assumption, together with Exercise 3.1.5 p.78 of Kunita's book [23], implies that $\hat{a}^{2}$ has a modification that is smooth enough in $(\tau, x)$. The third assumption guarantees the existence of the fundamental solution of (6) as a well-behaved random process in $t$. Finally, assumptions (4) and (5) are purely technical.

If we want to use the model specified above by a continuum of Itô's stochastic differentials for simulation purposes, we have to specify the stochastic spot volatility process $\sigma$, the random fields $\alpha$ and $\beta$, infer the values of $S_{0}$ and $\hat{a}_{0}^{2}$ from observed prices, and only then simulate the sample paths of underlying stock price $S_{t}$ and its local volatility surface $\hat{a}$ (which in turn will give us the sample paths of option prices). However, the main result of the paper is that if we want to avoid arbitrage, the only parameter to be specified is $\beta$, all the other specifications will follow.

\section{Semi-martingale Representation of the Option Prices}

The goal of this section is to show that for each fixed $\tau>0$ and $x \in \mathbb{R}$, the option price process $\hat{C}(\tau, x)$ defined for each fixed $t$, as the solution in $\tau$ and $x$ of the initial value problem (6) is a semimartingale in $t$. This yields the same result for $\tilde{C}(\tau, K)$ for $t>0$, and $C(T, K)$ for $t \in[0, T]$. These results are based on two auxiliary lemmas. Even though the results of these lemmas are expected to hold, especially under the smoothness assumptions made above, their proofs are quite lengthy and technical. So in order not to disrupt the flow of the paper, we state them without proof, postponing the gory details to appendices at the end of the paper.

Using the notation

$$
D_{x}:=\frac{1}{2} \partial_{x x}^{2}-\partial_{x} \quad \text { and } \quad L_{x}:=\hat{a}_{t}^{2}(\tau, x) D_{x}
$$

equation (6) becomes

$$
\left\{\begin{array}{l}
\partial_{\tau} \hat{C}(\tau, x)=L_{x} \hat{C}, \quad \tau>0, x \in \mathbb{R} \\
\hat{C}(0, x)=\left(S-e^{x}\right)^{+}
\end{array} .\right.
$$


Our first step is to smooth the initial condition with an approximate identity. We introduce $\varphi(x) \in$ $C_{0}^{\infty}(\mathbb{R})$ such that

(1) $\varphi(x) \geq 0$;

(2) $\varphi(x)=0$ for $|x| \geq 1$;

(3) $\int_{\mathbb{R}} \varphi(u) d u=1$;

(4) $\int_{-\infty}^{1} \int_{-\infty}^{x} \varphi(y) d y d x=1$.

Then we set

$$
F(x)=\int_{-\infty}^{x} \int_{-\infty}^{y} \varphi(z) d z d y
$$

and for each $\varepsilon>0$ we define $F_{\varepsilon}(x)$ by

$$
F_{\varepsilon}(x)=\varepsilon F\left(\frac{x}{\varepsilon}\right)
$$

so that $F_{0}(x)=x^{+}$. Now, consider the solution $\hat{C}^{\varepsilon}$ of the initial value problem

$$
\left\{\begin{array}{l}
\partial_{\tau} \hat{C}^{\varepsilon}=L_{x} \hat{C}^{\varepsilon}, \tau>0, x \in \mathbb{R} \\
\hat{C}^{\varepsilon}(0, x)=F_{\varepsilon}\left(S-e^{x}\right) .
\end{array}\right.
$$

The purpose of the next lemma is to prove that, as a process in $t$, the solution is a semi-martingale.

Lemma 1. There exist predictable processes $\left\{\hat{\mu}_{t}^{\varepsilon}(\tau, x)\right\}_{t}$ and $\left\{\hat{\nu}_{t}^{\varepsilon, i}(\tau, x)\right\}_{t}$ for $i=1, \cdots, m$ with values in $C^{1,2}\left(\mathbb{R}_{+} \times \mathbb{R}\right)$, such that for any $(t, \tau, x)$

$$
\hat{C}_{t}^{\varepsilon}(\tau, x)=\hat{C}_{0}^{\varepsilon}(\tau, x)+\int_{0}^{t} \hat{\mu}_{u}^{\varepsilon}(\tau, x) d u+\int_{0}^{t} \hat{\nu}_{u}^{\varepsilon}(\tau, x) \cdot d B_{u}
$$

holds $\mathbb{Q}$-a.s.

The proof of this result is given in the first part of Appendix A. Once we know that the prices $\hat{C}_{t}^{\varepsilon}(\tau, x)$ of options with smoothed pay-offs are semi-martingales, we show that, when $\varepsilon \searrow 0$, the semi-martingale property is preserved.

Lemma 2. There exist predictable processes $\left\{\hat{\mu}_{t}(\tau, x)\right\}_{t}$ and $\left\{\hat{\nu}_{t}^{i}(\tau, x)\right\}_{t}$ for $i=1, \cdots, m$ with values in $C^{1,2}\left(\mathbb{R}_{++} \times \mathbb{R}\right)$, such that for any $(t, \tau, x)$

$$
\hat{C}_{t}(\tau, x)=\hat{C}_{0}(\tau, x)+\int_{0}^{t} \hat{\mu}_{u}(\tau, x) d u+\int_{0}^{t} \hat{\nu}_{u}(\tau, x) \cdot d B_{u}
$$

holds $\mathbb{Q}$-a.s.

The proof is given in the second part of Appendix A. We conclude this section with two more technical results which will be needed in the proof of the main result of the paper.

Proposition 1. For any fixed $t>0$ and compact $\mathcal{K} \subset \mathbb{R}$ we have:

$$
\lim _{\tau \searrow 0} \mathbb{E} \int_{\mathcal{K}}\left(\int_{0}^{t}\left(\hat{\nu}_{u}(\tau, x)-\hat{\nu}_{u}(0, x)\right) \cdot d B_{u}\right)^{2} d x+\mathbb{E} \int_{\mathcal{K}}\left(\hat{C}_{t}(\tau, x)-\left(S_{t}-e^{x}\right)^{+}\right)^{2} d x=0 .
$$

The proof of this result is given in the first part of Appendix B. 
Proposition 2. $\mathbb{Q}$ - almost surely, for any $t>0$ and $h \in C_{0}^{\infty}(\mathbb{R})$, we have

$$
\lim _{\tau \searrow 0} \int_{\mathbb{R}} h(x) \hat{\mu}_{t}(\tau, x) d x=\frac{1}{2} S_{t} \sigma_{t}^{2} h\left(\log S_{t}\right) .
$$

The proof of this result is given in the second part of Appendix B.

\section{Absence of Arbitrage}

Since the stock price process is already assumed to be a local martingale under the pricing measure, in the present context, absence of arbitrage merely means that for each fixed strike $K>0$ and for each fixed date of maturity $T>0$, the option prices processes

$$
\left\{C_{t}(T, K)\right\}_{t \in[0, T)}=\left\{\hat{C}_{t}(T-t, x)\right\}_{t \in[0, T)}
$$

are local martingales. The following theorem addresses this problem. It is the main thrust of the paper.

Theorem 1. Absence of arbitrage is equivalent to the following conditions being satisfied a.s. for all $t>0, \tau>0, x \in \mathbb{R}$ :

Drift restriction:

$$
\hat{\alpha}_{t}+\frac{\frac{d}{d t}\left\langle\log \hat{a}_{t}^{2}, D_{x} \hat{C}_{t}\right\rangle}{D_{x} \hat{C}_{t}}=\partial_{\tau} \log \hat{a}_{t}^{2}
$$

\section{Volatility specification:}

$$
\hat{a}_{t}^{2}\left(0, \log S_{t}\right)=\sigma_{t}^{2}
$$

Remarks 1. The drift condition (8) can be expressed in a more convenient form, especially when it comes to numerical implementation:

$$
\hat{\alpha}+\frac{\hat{\beta} \cdot D_{x} \hat{\nu}}{D_{x} \hat{C}}=\partial_{\tau} \log \hat{a}^{2}
$$

where $\hat{\nu}$ is the volatility in the semi-martingale decomposition of the call price process, as given for example by the solution of the system of partial differential equations (48).

2. We can also rewrite (8) in the way it was originally touted by Derman and Kani in [12]. If we plug in (8) the representation of $\hat{\nu}(\tau, x)$ in terms of $\hat{p}$ and $\hat{q}$ (the fundamental solutions of (5) and (42) respectively), we obtain

$$
\begin{gathered}
\hat{\alpha}(\tau, x)+\frac{\sum_{i=1}^{m} \hat{\beta}^{i}(\tau, x) \int_{\mathbb{R}} \int_{0}^{\tau} \hat{a}^{2}(u, y) \hat{\beta}^{i}(u, y) \hat{q}(0, \log S ; u, y) D_{y} \hat{q}(u, y ; \tau, x) d u d y}{\hat{q}(0, \log S ; \tau, x)} \\
+S \sigma \frac{\hat{\beta}^{1}(\tau, x) \partial_{S} \hat{q}(0, \log S ; \tau, x) d y}{\hat{q}(0, \log S ; \tau, x)}=\partial_{\tau} \log \hat{a}^{2}(\tau, x) .
\end{gathered}
$$

This is essentially, after changing variables from the couple ("maturity","strike") used by Derman and Kani in [12], to the couple ("time-to-maturity","log-strike") used here, the drift condition proposed by Derman and Kani. However, the third term of the above left hand side seems to be missing in [12]! 
First, we notice that (8) is well defined since $D_{x} \hat{C}$ stays positive because of the maximum principle applied to the fundamental solution of a uniformly parabolic initial value problem. From the generalized Itô rule (see for example Theorem 3.3.1 of [23]) we know that, for any $T>0$ and $x \in \mathbb{R}$, the process $\left\{\hat{C}_{t}(T-t, x)\right\}_{t \in[0, T)}$ is a semi-martingale with the following decomposition

$$
d \hat{C}_{t}(T-t, x)=\left(\hat{\mu}_{t}(T-t, x)-\partial_{\tau} \hat{C}_{t}(T-t, x)\right) d t+\hat{\nu}_{t}(T-t, x) \cdot d B_{t}
$$

Proof of the "only if" part. Assume that $\left\{\hat{C}_{t}(T-t, x)\right\}_{t \in[0, T)}$ is a martingale for every fixed $T>0$ and $x \in \mathbb{R}$. Then

$$
d \hat{C}_{t}(T-t, x)=\hat{\nu}_{t}(T-t, x) \cdot d B_{t}
$$

and, from the generalized Itô formula

$d\left(\hat{a}_{t}^{2}(T-t, x)\right)=\left(\hat{a}_{t}^{2}(T-t, x) \hat{\alpha}_{t}(T-t, x)-\partial_{\tau} \hat{a}_{t}^{2}(T-t, x)\right) d t+\hat{a}_{t}^{2}(T-t, x) \hat{\beta}_{t}(T-t, x) \cdot d B_{t}$

Recall that, because of the defining relationship between local volatility and call prices we have a.s.

$$
\hat{a}_{t}^{2}(T-t, x) \cdot\left(\partial_{x x}^{2}-\partial_{x}\right) \hat{C}_{t}(T-t, x)=2 \partial_{\tau} \hat{C}_{t}(T-t, x),
$$

and from Exercise 3.1.5, 78, of Kunita's book [23], we know that $\left(\partial_{x x}^{2}-\partial_{x}\right) \hat{C}_{t}(T-t, x)$ and $\partial_{\tau} \hat{C}_{t}(T-t, x)$ are local martingales as processes in $t$. Therefore, the drift on the right side of (11) is zero. Writing that the left side is zero as well we get:

$$
\begin{aligned}
d\left(\hat{a}_{t}^{2}(T-t, x)\right. & \left.\cdot D_{x} \hat{C}_{t}(T-t, x)\right)_{t} \\
=\left(\hat{a}_{t}^{2}(T-t, x) \hat{\alpha}_{t}(T-t, x)-\partial_{\tau} \hat{a}_{t}^{2}(T-t, x)\right) D_{x} \hat{C}_{t}(T-t, x) d t & \\
& \quad+\left(\frac{\partial}{\partial t}\left\langle\hat{a}_{t}^{2}(T-t, x), D_{x} \hat{C}_{t}(T-t, x)\right\rangle\right) d t+(\ldots) \cdot d B_{t}
\end{aligned}
$$

This yields (8). Now, fix some test function $h \in C_{0}^{\infty}(\mathbb{R})$, denote by $\Lambda(y)$ the local time of $S$ at $y$, and, from Proposition 1, we have:

$$
\begin{aligned}
& \left(\mathbb{E} \int_{\text {supp }(h)}\left(\int_{0}^{t} \hat{\mu}_{u}(\tau, x) d u-\Lambda\left(e^{x}\right)\right)^{2} d x\right)^{1 / 2} \\
& =\left(\mathbb{E} \int_{\operatorname{supp}(h)}\left(\hat{C}_{t}(\tau, x)-\left(S_{t}-e^{x}\right)^{+}-\int_{0}^{t}\left(\hat{\nu}_{u}(\tau, x)-\hat{\nu}_{u}(0, x)\right) \cdot d B_{u}\right)^{2} d x\right)^{1 / 2} \\
& \leq\left(\mathbb{E} \int_{\operatorname{supp}(h)}\left(\int_{0}^{t}\left(\hat{\nu}_{u}(\tau, x)-\hat{\nu}_{u}(0, x)\right) \cdot d B_{u}\right)^{2} d x\right)^{1 / 2} \\
& +\left(\mathbb{E} \int_{\operatorname{supp}(h)}\left(\hat{C}_{t}(\tau, x)-\left(S_{t}-e^{x}\right)^{+}\right)^{2} d x\right)^{1 / 2}
\end{aligned}
$$

which tends to 0 as $\tau \searrow 0$. From this we conclude that there exists a sequence $\tau_{n} \rightarrow 0$, such that almost surely

$$
\lim _{n \rightarrow \infty} \int_{\text {supp }(h)}\left(\int_{0}^{t} \hat{\mu}_{u}\left(\tau_{n}, x\right) d u-\Lambda_{t}\left(e^{x}\right)\right)^{2} d x=0
$$


Recall that, since $\left(\hat{C}_{t}(T-t, x)\right)_{t \in[0, T)}$ is a local martingale for any $T>0$, its drift is zero. So using (10), we can conclude that

$$
\hat{\mu}_{u}\left(\tau_{n}, x\right)=\partial_{\tau} \hat{C}_{u}\left(\tau_{n}, x\right)=\frac{1}{2} \hat{a}_{u}^{2}\left(\tau_{n}, x\right) D_{x} \hat{C}_{u}\left(\tau_{n}, x\right),
$$

and from (12) and (13) we have, almost surely for any $t$ and $h \in C_{0}^{\infty}(\mathbb{R})$

$$
\begin{aligned}
0 & =\lim _{n \rightarrow \infty} \int_{\mathbb{R}} h(x)\left(\Lambda_{t}\left(e^{x}\right)-\left(\frac{1}{2} \int_{0}^{t} \hat{a}_{u}^{2}\left(\tau_{n}, x\right) D_{x} \hat{C}_{u}\left(\tau_{n}, x\right) d u\right) d x\right) \\
& =\int_{\mathbb{R}} h(x) \Lambda_{t}\left(e^{x}\right) d x-\frac{1}{2} \lim _{n \rightarrow \infty}\left(\int_{0}^{t} \int_{\mathbb{R}} h(x) \hat{a}_{u}^{2}\left(\tau_{n}, x\right) D_{x} \hat{C}_{u}\left(\tau_{n}, x\right) d x d u\right) .
\end{aligned}
$$

Now, using the definition of the local time, and dominated convergence, we get

$$
\begin{gathered}
\frac{1}{2} \int_{0}^{t} h\left(\log S_{u}\right) S_{u} \sigma_{u}^{2} d u-\frac{1}{2} \int_{0}^{t} \lim _{n \rightarrow \infty}\left(\int_{\mathbb{R}} h(x) \hat{a}_{u}^{2}\left(\tau_{n}, x\right) D_{x} \hat{C}_{u}\left(\tau_{n}, x\right) d x\right) d u \\
=\frac{1}{2} \int_{0}^{t} h\left(\log S_{u}\right) S_{u} \sigma_{u}^{2}-h\left(\log S_{u}\right) S_{u} \hat{a}_{u}^{2}\left(0, \log S_{u}\right) d u
\end{gathered}
$$

which holds for any $t>0$. The above integrand is a.s. continuous, therefore, it is identically equal to zero. And since this is true for any $h \in C_{0}^{\infty}(\mathbb{R})$, we conclude that $\hat{a}_{t}^{2}\left(0, \log S_{t}\right)=\sigma_{t}^{2}$ which completes the proof of (9).

Proof of the "if" part. From Lemma 1 and Lemma 2 of the Section 3 above, we know that $\left\{\hat{C}_{t}(T-\right.$ $t, x)\}_{t \in[0, T)}$ is an Itô process. Let us denote its drift at time $t$ by $\hat{v}_{t}(T-t, x)$. Smoothness of $\hat{C}_{t}(\tau, x)$, $\hat{\mu}_{t}(\tau, x)$ and $\hat{\nu}_{t}(\tau, x)$ yields that $\hat{v}_{t}(\tau, x) \in C^{1,2}\left(\mathbb{R}_{++} \times \mathbb{R}_{+}\right)$. Then, from (8), by differentiating (11) with respect to $t$ (just like it was done in the first part of the proof), we obtain:

$$
\partial_{\tau} \hat{v}_{t}(\tau, x)=\frac{1}{2} \hat{a}_{t}^{2}(\tau, x) D_{x} \hat{v}_{t}(\tau, x), \quad \tau>0, x \in \mathbb{R}
$$

which holds almost surely for all $t>0$. Since

$$
\hat{v}_{t}(\tau, x)=\hat{\mu}_{t}(\tau, x)-\partial_{\tau} \hat{C}_{t}(\tau, x)
$$

for any test function $h \in C_{0}^{\infty}(\mathbb{R})$ we have

$$
\int_{\mathbb{R}} h(x) \hat{v}_{t}(\tau, x) d x=\int_{\mathbb{R}} h(x) \hat{\mu}_{t}(\tau, x) d x-\int_{\mathbb{R}} h(x) \partial_{\tau} \hat{C}_{t}(\tau, x) d x
$$

As $\tau \rightarrow 0$, the first term in the above right hand side converges to $\frac{1}{2} S_{t} \sigma_{t}^{2} h\left(\log S_{t}\right)$ because of Proposition 2 of Section 3 above. Moreover

$$
\begin{aligned}
\int_{\mathbb{R}} h(x) \partial_{\tau} \hat{C}_{t}(\tau, x) d x & =\frac{1}{2} \int_{\mathbb{R}} h(x) \hat{a}_{t}^{2}(\tau, x) e^{x} \hat{q}_{t}\left(0, \log S_{t} ; \tau, x\right) d x \\
& \rightarrow \frac{1}{2} S_{t} h\left(S_{t}\right) \hat{a}_{t}^{2}\left(0, S_{t}\right) \\
& =\frac{1}{2} S_{t} \sigma_{t}^{2} h\left(\log S_{t}\right) .
\end{aligned}
$$


This proves that, almost surely for any $t, \hat{v}_{t}(\tau, x)$, as function of $x$, converges weakly to 0 as $\tau \rightarrow 0$. Then, from the uniqueness of the weak solution of (14) (see for example, [13]), we conclude that, for any $\tau>0, \hat{v}_{t}(\tau, \cdot) \equiv 0$ in $\mathrm{七}_{l o c}^{2}(\mathbb{R})$ sense. But since $\hat{v}_{t}(\tau, x)$ is continuous, it is equal to zero for any $\tau>0$ and $x \in \mathbb{R}$.

This implies that $\left(\hat{C}_{t}(T-t, x)\right)_{t \in[0, T)}$ is a local martingale in $t$ for any $T>0$ and $x \in \mathbb{R}$. But since $\hat{C}_{t} \leq S_{t}$, it is square integrable, and therefore every $\left(\hat{C}_{t}(T-t, x)\right)_{t \in[0, T)}$ is a martingale. This completes the proof of the theorem.

Implementation. We are now in a position to give the specifics of the pricing Monte Carlo algorithm introduced earlier:

(1) The last theorem shows that the only free parameter in the model is $\left\{\hat{\beta}_{t}(\tau, K)\right\}_{t \geq 0}$. It is chosen from intuition and historical observations not already contained in the set of observed call option prices.

(2) We use the current stock price for $S_{0}$, and the call option prices to deduce the initial local volatility surface. Many methods have been proposed to do that, ranging from parametric and non-parametric statistical methods (see for example [17] and the references therein) and smoothing by optimization and partial differential equations (see for example [1]). We revisit this issue later in the paper.

(3) We simulate sample paths of $S_{t}$ and $\hat{a}_{t}^{2}$ as follows. Given $\hat{a}_{t}^{2}$ and $\hat{\beta}_{t}$, we compute $\hat{\alpha}_{t}$ from the no-arbitrage condition (8) and $\sigma_{t}$ from the consistency condition (9). Then we use $\hat{\alpha}_{t}$ and $\hat{\beta}_{t}$ to find $\hat{a}_{t+\Delta t}^{2}$ and use $\sigma_{t}$ to find $S_{t+\Delta t}$ by a single step in a forward plain Euler's scheme, and we iterate. Voila!

This algorithm will give us arbitrage-free dynamics of the option prices together with the stock. It will allow us to calculate the prices of other derivatives that use the stock and/or the dynamically modeled option prices as underlying.

\section{EXAMPLES}

This section provides a couple of simple consequences of the no-arbitrage condition derived above.

5.1. Bounded Variations Dynamics and Markovian Spot Models. Let us first consider the simplest possible model of local volatility stochastic dynamics by assuming that $\left\{\hat{\beta}_{t}(\tau, x)\right\}_{t \geq 0}$ is identically zero, i.e. $\hat{\beta} \equiv 0$. Obviously, this corresponds to assuming that the time evolution of the local volatility surface is of bounded variations. Under this assumption, the no-arbitrage drift condition (8) becomes

$$
\partial_{\tau} \hat{\alpha}_{t}(\tau, x)=\hat{\alpha}_{t}(\tau, x)=\partial_{\tau} \log \tilde{a}_{t}^{2}(\tau, x)
$$

which, for each fixed strike $K=e^{x}$, is a plain hyperbolic transport equation whose solutions are given by traveling waves. Consequently

$$
\hat{a}_{t}^{2}(\tau, x)=\hat{a}_{0}^{2}(\tau+t, x)
$$

from which we conclude, using the consistency condition (9), that

$$
\sigma_{t}=\hat{a}_{0}\left(t, S_{t}\right) .
$$

This above derivation proves the following aside: 
Proposition 3. The local volatility surface is a process of bounded variation if and only if it is the shift of a fixed deterministic surface along the space-time underlying sample path, and the underlying stock process is Markovian.

5.2. Case of Flat Surfaces. Let us now assume that the local volatility surface is only driven by the second component of the Brownian motion, and from some instant of time $t$ on, $\hat{a}_{t}^{2}(\tau, x)$ and its diffusion term $\hat{\beta}_{t}^{2}(\tau, x)$ become flat (constant functions $\hat{a}_{t}^{2}$ and $\hat{\beta}_{t}^{2}$ of $\tau$ and $x$ ). Then we can solve (6) and (48) explicitly, and the no-arbitrage drift condition (8) gives the following expression for the drift

$$
\hat{\alpha}_{t}(\tau, x)=-\left\|\hat{\beta}_{t}\right\|^{2}\left(\frac{\left(x-\log S_{t}\right)^{2}}{\hat{a}_{t}^{2}}-1-\sqrt{\tau}\right) .
$$

This is another instance of the fact that in the absence of arbitrage, the drift $\hat{\alpha}$ is determined by the volatility structure $\hat{\beta}$.

5.3. Random Scaling. Here we consider the case of a local volatility surface obtained by randomly scaling a fixed deterministic surface.

Proposition 4. If $\hat{a}_{t}^{2}(\tau, x)=\lambda_{t} \hat{a}_{0}^{2}(\tau, x)$ for some semi-martingale $\lambda$ with the decomposition $d \lambda_{t}=$ $\alpha_{t} d t+\beta_{t} \cdot d B_{t}$, and some fixed deterministic initial surface $\hat{a}_{0}^{2}$, then absence of arbitrage implies that $\lambda$ is a deterministic function of the underlying stock.

In such a case, the setting of the proposition reduces to the simple constant local volatility model discussed above. For the proof we assume in addition that $\hat{a}_{0}^{2} \in C^{3}\left(\mathbb{R}^{2}\right)$, and that for any $c>0$, the order of growth of its derivatives is not more than $e^{c x^{2}}$, on some strip $[0, T] \times \mathbb{R}$.

If we multiply both sides of the no-arbitrage drift condition (8) by $D_{x} \hat{C}$ and integrate with respect to $x$, then the second term on the left side disappears since $\int_{\mathbb{R}} D_{x} \hat{\nu} d x=0$. This leaves us with:

$$
\hat{\alpha}=\int_{\mathbb{R}} \partial_{\tau} \log \hat{a}_{0}^{2}(\tau, x) \hat{q}(0, \log S ; \tau, x) d x
$$

and differentiating both sides with respect to $\tau$ and using the properties of the fundamental solution, we obtain

$\int_{\mathbb{R}} \partial_{\tau \tau} \log \hat{a}_{0}^{2}(\tau, x) \hat{q}_{t}(0, \log S ; \tau, x) d x+\int_{\mathbb{R}} \partial_{\tau} \log \hat{a}_{0}^{2}(\tau, x) D_{x}\left(\lambda_{t} \hat{a}_{0}^{2}(\tau, x) \hat{q}_{t}\left(0, \log S_{t} ; \tau, x\right)\right) d x=0$.

Integrating by parts the second term and letting $\tau \searrow 0$ we get:

$$
\partial_{\tau \tau} \log \hat{a}_{0}^{2}\left(0, \log S_{t}\right)+\lambda_{t} \hat{a}_{0}^{2}\left(0, \log S_{t}\right) D_{x}^{*} \partial_{\tau} \log \hat{a}_{0}^{2}\left(0, \log S_{t}\right)=0
$$

which proves that, excluding some degenerate cases, $\lambda_{t}$ is a deterministic function of the underlying.

\section{From Stochastic to Local Volatility: The Markovian Case}

Although it is clear that all market models in which European call prices are semi-martingales (sufficiently smooth in strike and maturity) are included in our framework, it is interesting to see how the local volatility looks in some of the most popular models.

We consider stochastic volatility models in which the volatility process is Markovian with respect to its own filtration and given by an Itô stochastic differential equation. Obviously, the Hull-White 
and Heston models are particular cases. Our goal is to exhibit generic properties of the local volatility surface in these models. So we assume that

$$
\left\{\begin{array}{l}
d S_{t}=S_{t} r d t+S_{t} \sigma_{t}\left(\sqrt{1-\rho^{2}} d B_{t}^{1}+\rho d B_{t}^{2}\right), \quad S_{0} \\
d \sigma_{t}=f\left(t, \sigma_{t}\right) d t+g\left(t, \sigma_{t}\right) d B_{t}^{2}, \quad \sigma_{0}
\end{array}\right.
$$

where $\left\{B_{t}^{1}\right\}_{t \geq 0}$ and $\left\{B_{t}^{2}\right\}_{t \geq 0}$ are independent Brownian motions, $\rho \in[-1,+1]$, and $f(t, x)$ and $g(t, x)$ satisfy the usual conditions which guarantee existence and uniqueness of a positive solution to the above system. Notice that, contradictory to the convention used in this paper, we featured the interest rate $r$ in the model. This is to emphasize its role (or lack thereof) in the formula we derive in this section.

Notice also that, for the purpose of this section we can limit ourselves to the case $t=0$, and doing so we are back to the static case considered originally by Dupire and Derman and Kani, and we can use $\tau$ or $T$ interchangeably, ignoring the tildes and hats on the coefficients. The goal of this section is to state and prove a new expression for the local volatility of Markovian stochastic volatility models.

Proposition 5. In a stochastic volatility model (16), the local volatility surface is given at time $t=0$ by the formula:

$$
a^{2}(T, K)=\frac{\mathbb{E}\left[\frac{\bar{S}_{T} \sigma_{T}^{2}}{\bar{\sigma}_{T}} e^{-\frac{d_{1}^{2}(T, K)}{2}}\right]}{\mathbb{E}\left[\frac{\bar{S}_{T}}{\bar{\sigma}_{T}} e^{-\frac{d_{1}^{2}(T, K)}{2}}\right]}
$$

where $\bar{S}_{T}, \bar{\sigma}_{T}$ and $d_{1}(T, x)$ are defined in formulae (18), (19) and (22) below.

Proof:

Solving the system (16) for $S_{t}$ we get:

$$
\begin{aligned}
S_{t}=S_{0} \exp ( & \left.-\rho \int_{0}^{t} \sigma_{u} \frac{f\left(u, \sigma_{u}\right)}{g\left(u, \sigma_{u}\right)} d u+\rho \int_{0}^{t} \frac{\sigma_{u}}{g\left(u, \sigma_{u}\right)} d \sigma_{u}\right) \\
& \quad \exp \left(r t-\frac{1}{2} \int_{0}^{t} \sigma_{u}^{2} d u+\sqrt{\left(1-\rho^{2}\right)} \int_{0}^{t} \sigma_{u} d B_{u}^{1}\right) .
\end{aligned}
$$

For each $t>0$ we define the quantities $\bar{S}_{t}$ and $\bar{\sigma}_{t}$ by

$$
\bar{S}_{t}=S_{0} \exp \left(-\frac{\rho^{2}}{2} \int_{0}^{t} \sigma_{u}^{2} d u-\rho \int_{0}^{t} \sigma_{u} \frac{f\left(u, \sigma_{u}\right)}{g\left(u, \sigma_{u}\right)} d u+\rho \int_{0}^{t} \frac{\sigma_{u}}{g\left(u, \sigma_{u}\right)} d \sigma_{u}\right)
$$

and

$$
\bar{\sigma}_{t}=\sqrt{\frac{1}{t} \int_{0}^{t} \sigma_{u}^{2} d u} .
$$

Both quantities depend only on $\left\{B_{u}^{2}\right\}_{0 \leq u \leq t}$. Moreover,

$$
\begin{aligned}
C(T, K) & =\mathbb{E}\left\{\left(S_{T}-K\right)^{+}\right\} \\
& =\mathbb{E}\left\{\left(\bar{S}_{T} \exp \left(r T-\frac{\left(1-\rho^{2}\right)}{2} T \bar{\sigma}_{T}^{2}+\sqrt{\left(1-\rho^{2}\right)} \int_{0}^{T} \sigma_{u} d B_{u}^{1}\right)-K\right)^{+}\right\}
\end{aligned}
$$


so that, conditioning by $\left\{B_{u}^{2}\right\}_{0 \leq u \leq t}$, we obtain

$$
C(T, K)=\mathbb{E}\left\{B S\left(\bar{S}_{t}, T, \sqrt{\left(1-\rho^{2}\right)} \bar{\sigma}_{T}, K\right)\right\}
$$

where the expectation is over the second Brownian motion $\left\{B_{t}^{2}\right\}_{t \geq 0}$, and where the Black-Scholes call price $B S(S, T, \sigma, K)$ was defined in (2). Itô's rule gives

$$
\begin{gathered}
d B S\left(\bar{S}_{t}, T, \sqrt{\left(1-\rho^{2}\right)} \bar{\sigma}_{T}, K\right)=\Phi\left(d_{1}\right) \bar{S}_{T} \rho \sigma_{T} d B_{T}^{2}-r K e^{-r T} \Phi\left(d_{1}-\sqrt{T} \sqrt{1-\rho^{2}} \bar{\sigma}_{T}\right) d T \\
+\frac{1}{\sqrt{1-\rho^{2}}} \frac{\bar{S}_{T} \sigma_{T}^{2}}{\bar{\sigma}_{T}} \frac{1}{\sqrt{T}} \frac{1}{2 \sqrt{2 \pi}} e^{-\frac{d_{1}^{2}}{2}} d T
\end{gathered}
$$

where $d_{1}=d_{1}(T, K)$ is defined in the usual way by:

$$
d_{1}(T, K)=\frac{\log \frac{\bar{S}_{T}}{K}+\left(r+\frac{1}{2}\left(1-\rho^{2}\right) \bar{\sigma}_{T}^{2}\right) T}{\sqrt{1-\rho^{2}} \bar{\sigma}_{T} \sqrt{T}} .
$$

Taking expectations on both sides and using Fubini's theorem we obtain

$$
\partial_{T} C(T, K)=-r K e^{-r T} \mathbb{E}\left[\Phi\left(d_{1}-\sqrt{T} \sqrt{1-\rho^{2}} \bar{\sigma}_{T}\right)\right]+\frac{1}{\sqrt{1-\rho^{2}}} \frac{1}{\sqrt{T}} \frac{1}{2 \sqrt{2 \pi}} \mathbb{E}\left[\frac{\bar{S}_{T} \sigma_{T}^{2}}{\bar{\sigma}_{T}} e^{-\frac{d_{1}^{2}}{2}}\right] .
$$

Repeated differentiations and uses of Fubini's theorem give

$$
\partial_{K} C(T, K)=-e^{-r T} \mathbb{E}\left[\Phi\left(d_{1}-\sqrt{T} \sqrt{1-\rho^{2}} \bar{\sigma}_{T}\right)\right]
$$

and

$$
\partial_{K K} C(T, K)=\frac{1}{\sqrt{2 \pi} \sqrt{1-\rho^{2}} \sqrt{T} K^{2}} \mathbb{E}\left[\frac{\bar{S}_{T}}{\bar{\sigma}_{T}} e^{-\frac{d_{1}^{2}}{2}}\right]
$$

which in turn gives the desired result since:

$$
a^{2}(T, K)=\frac{2\left(\partial_{T} C+r K \partial_{K} C\right)}{K^{2} \partial_{K K} C}
$$

Notice that formula (17) still contains expectations. However, these expectations are only over the paths of the stochastic volatility $\sigma_{t}$ (i.e. over the second Brownian motion $\left\{B_{t}^{2}\right\}_{t \geq 0}$ ), and in order to implement this formula in Monte Carlo computations, we only need to simulate the (Markovian) paths of $\sigma_{t}$. But the main strength of formula (17) is to have rid of the singularities in the numerator and denominator. Such singularities would appear automatically if we were to try to use straightforward Monte Carlo simulations. Finally, we notice that the expectations appearing in formula (17) can be computed explicitly if the joint distribution of $\left(\sigma_{t}, \int_{0}^{t} \sigma_{u}^{2} d u\right)$ is known. This is for example the case in Heston and Hull-White models.

Figure 1 gives the plot of a typical local volatility surface in Heston's model. It was computed by Monte Carlo evaluation of the expectations appearing in formula (17). Artifacts due to inaccuracies in the Monte Carlo evaluations can be seen on both sides of the surface. 


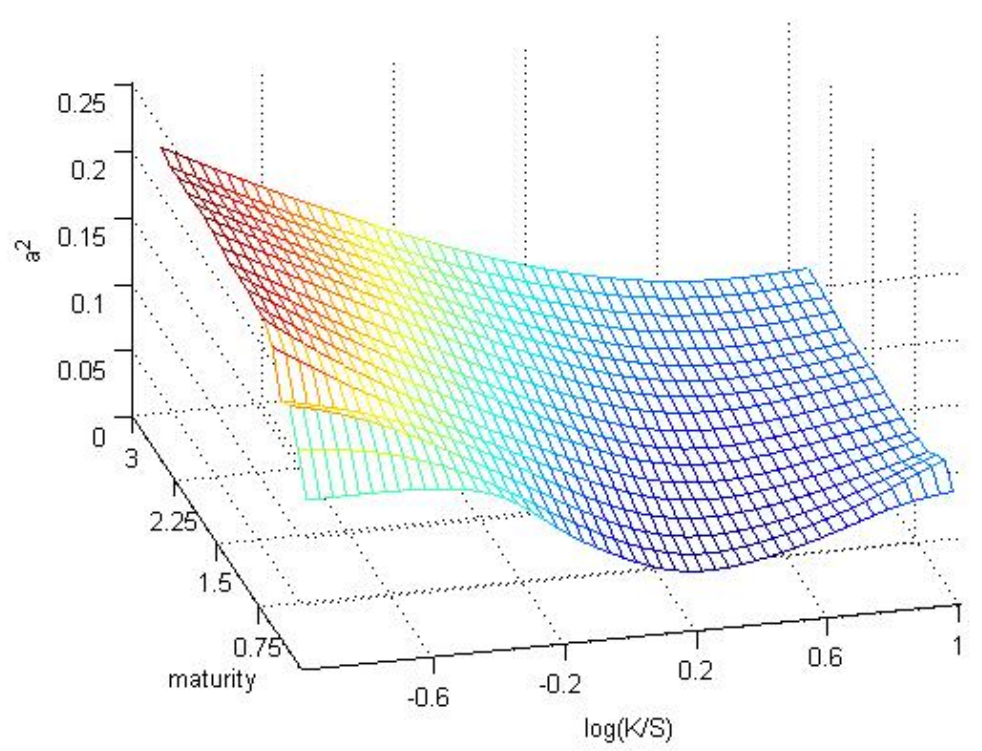

FIGURE 1. Typical local volatility surface in Heston's Model

We used the values $\mu=0.045, \sigma_{0}=0.3, \hat{\sigma}=0.2, k=0.23, \theta=0.2$ and $\rho=-0.5$ in the stochastic system

$$
\left\{\begin{array}{cc}
d S_{t}=\mu S_{t} d t+S_{t} \sigma_{t}\left(\sqrt{1-\rho^{2}} d B_{t}^{1}+\rho d B_{t}^{2}\right), & S_{0} \\
d \sigma_{t}^{2}=k\left(\theta-\sigma_{t}^{2}\right) d t+\hat{\sigma} \sigma_{t} d B_{t}^{2}, & \sigma_{0}
\end{array}\right.
$$

\section{A Parametric Family of Local Volatility Surfaces}

Several non-parametric methods have been proposed to fit local volatility surfaces to actual option data. See for example the books [1] by Achdou and Pironneau, and [17] by Fengler, and the references therein. Building on the intuition developed in the previous section, we propose a simple parametric family of surfaces for the same purpose of fitting a local volatility surface to observed market data. Our family is parameterized by the 9 scalar parameters

$$
\Theta=\left(\sigma, \eta_{1}, \eta_{2}, \theta_{1}, \theta_{2}, p_{1}, p_{2}, s, \mu\right)
$$

satisfying the conditions

$$
\begin{gathered}
p_{1}, p_{2} \geq 0, \quad p_{1}+p_{2} \leq 1 \\
\theta_{1}, \theta_{2} \geq 0 \\
\sigma>0, \quad \mu \geq 0 .
\end{gathered}
$$


We shall give the intuitive meaning of these parameters shortly. In the meantime, we introduce the notation, for $i=1,2$

$$
v_{i}(\tau)=\sqrt{\theta_{i}+\left(\sigma^{2}-\theta_{i}\right) \frac{1-e^{-\eta_{i} \tau}}{\eta_{i} \tau}}, \quad d_{i}(\tau, x)=\frac{s-x+\left(\mu+\frac{1}{2} v_{i}^{2}(\tau)\right) \tau}{\sqrt{\tau} v_{i}(\tau)},
$$

and

$$
\eta_{0}=0, p_{0}=1-p_{1}-p_{2}, v_{0}(\tau)=\sigma, d_{0}(\tau, x)=\frac{s-x+\left(\frac{1}{2} \sigma^{2}+\mu\right) \tau}{\sigma \sqrt{\tau}}
$$

We introduce the following collection of two-dimensional surfaces

$$
\hat{a}^{2}(\Theta, \tau, x)=\frac{\sum_{i=0}^{2} p_{i}\left(\theta_{i}+\left(\sigma^{2}-\theta_{i}\right) e^{-\eta_{i} \tau}\right) \exp \left(-\frac{d_{i}^{2}(\tau, x)}{2}\right) / v_{i}(\tau)}{\sum_{i=0}^{2} p_{i} \exp \left(-\frac{d_{i}^{2}(\tau, x)}{2}\right) / v_{i}(\tau)}
$$

The meaning of each of the parameters is as follows:

- $s$ is the logarithm of the current stock price;

- $\sigma$ is the spot volatility;

- $\mu$ is the drift of the stock process (most likely, the difference between interest rate and the dividends payment rate);

- $\left\{\eta_{i}, \theta_{i}\right\}_{i=1}^{2}$ define scenarios for the volatility process;

- $p_{i}$ 's are the respective probabilities of these scenarios.

The parametric family considered in this section is not new. Local volatility surfaces of the form (25) were introduced by Brigo and Mercurio in [3], [4], and by Brigo and collaborators in [31]. They described the local volatility surface corresponding to a stochastic volatility model in which the volatility process is independent of the Brownian motion driving the dynamics of the stock price, and can only take values in a finite set of deterministic functions $\sigma_{1}(t), \sigma_{2}(t), \ldots, \sigma_{m}(t)$, with probabilities $p_{1}, \ldots, p_{m}$. In this setting, clearly, the price of a European call option given by such a local volatility surface is

$$
C(\Theta, \tau, x)=\sum_{i=1}^{m} p_{i} B S\left(e^{s}, e^{x}, \tau, \sqrt{\frac{1}{\tau} \int_{0}^{\tau} \sigma_{i}^{2}(u) d u}, \mu\right) .
$$

A family of this type with 3 constant volatility functions $\sigma_{i}(t)$ was considered in [6]. But because such surfaces are singular as $\tau \searrow 0$, we consider here a different form of stochastic volatility sample paths in this paper. We choose

$$
\sigma_{i}^{2}(t)=\theta_{i}+\left(\sigma^{2}-\theta_{i}\right) e^{-\eta_{i} t}, i=0,1,2 .
$$

The motivation for this particular choice comes from our analysis of the Heston model in (23) with parameters $k$ and $\theta$ being random variables on a probability space $\Omega$ with three elements, $\Omega=$ $\left\{\omega_{0}, \omega_{1}, \omega_{2}\right\}$, independent of $\left(W^{1}, W^{2}\right)$, with $\mathbb{Q}\left(\omega_{1}\right)=p_{1}, \mathbb{Q}\left(\omega_{1}\right)=p_{2}$, and $k\left(\omega_{i}\right)=\eta_{i}, \theta\left(\omega_{i}\right)=$ $\theta_{i}, i=0,1,2$. In this way, any surface from the proposed parametric family can be interpreted as a limit of local volatility surfaces in the modified Heston model with $\hat{\sigma} \rightarrow 0$.

Figure 2 gives the graph of a local volatility surface from the above family. It was obtained by least squares fitting to the European call option prices quoted on the SP500 index on April 3rd, 2006. The 
parameter values produced by our least squares optimization are: $\sigma=0.16, \eta_{1}=0.26, \eta_{2}=0.21$, $\theta_{1}=0.52, \theta_{2}=0, p_{1}=0.26, p_{2}=0.31$ and $\mu=0.045$.

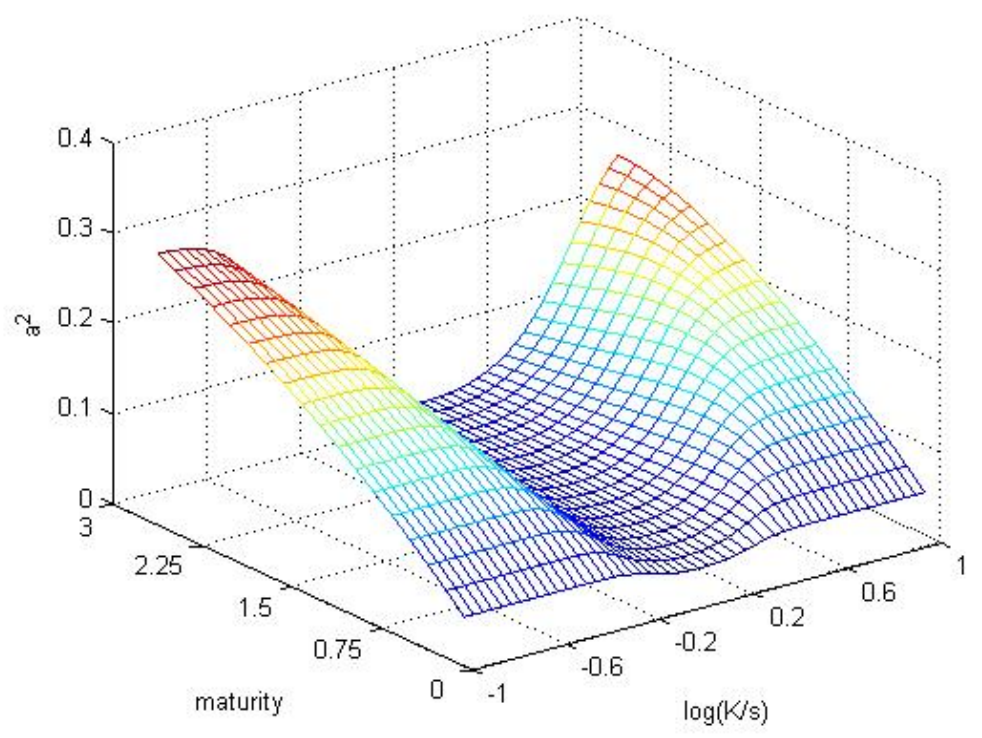

FIGURE 2. Local volatility surface from the parametric family introduced in this section. It was fitted to S\&P500 option prices on April 3rd 2006.

For the sake of comparison we give in Figure 3, the plot of a local volatility surface from a Heston model with $\rho=0$. Obviously, both local volatilities surfaces are monotone increasing with time to maturity, and as functions of $x$, they are $U$-shaped and flattening at infinity. 


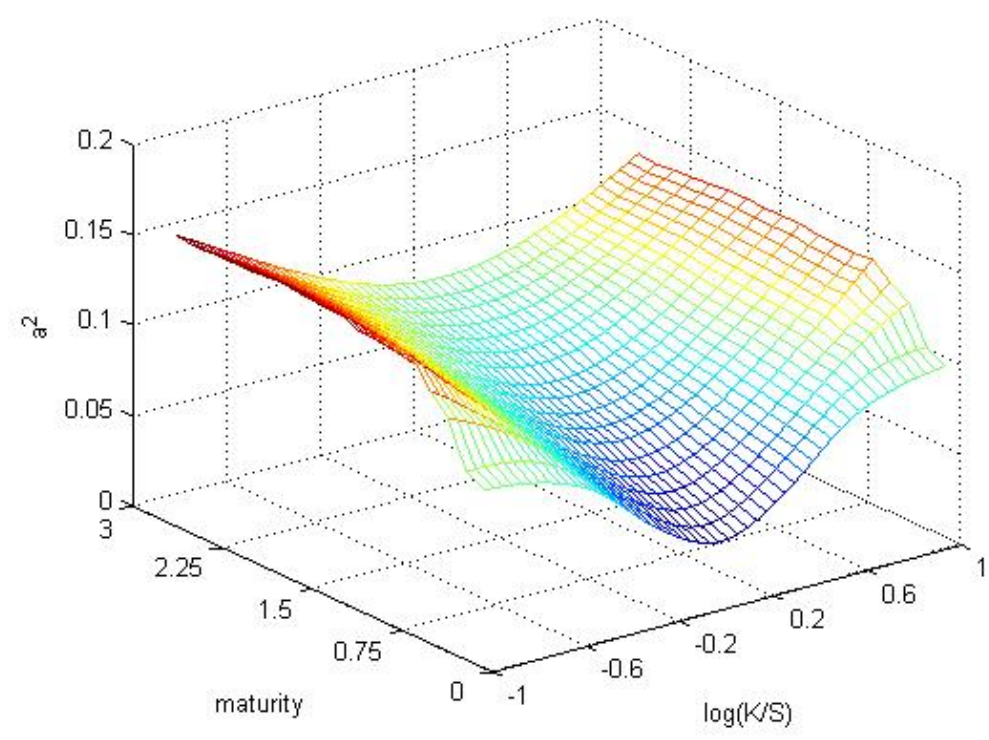

FIGURE 3. Local volatility surface of a Heston model with $\rho=0$ and the same parameters as those obtained from the least squares fit to S\&P 500 data above.

In order to introduce the dynamics of the local volatility surface, and also for the hedging purposes discussed in the next section, we need to estimate $\hat{\beta}(\tau, x)$. With this goal in mind, we propose a parametric form for $\hat{\beta}(\tau, x)$. Recall (25), and notice that if we pretend that the stochastic volatility model producing the local volatility (25) is true, the process $\sigma$ has almost surely finite variation. Then, formally using Itô's rule to compute $\left.d \hat{a}_{t}^{2}(\Theta, \tau, x)\right|_{t=0}$, we obtain

$$
\hat{\beta}(\Theta, \tau, x)=\frac{\sum_{i=0}^{2} p_{i} \frac{\hat{a}^{2}(\Theta, \tau, x)-\theta_{i}-\left(\sigma^{2}-\theta_{i}\right) e^{-\eta_{i} \tau}}{\sqrt{\tau} v_{i}(\tau) \hat{a}^{2}(\Theta, \tau, x)} d_{i}(\tau, x) \exp \left(-\frac{d_{i}^{2}(\tau, x)}{2}\right) / v_{i}(\tau)}{\sum_{i=0}^{2} p_{i} \exp \left(-\frac{d_{i}^{2}(\tau, x)}{2}\right) / v_{i}(\tau)}
$$

which gives us a parametric family for $\hat{\beta}(\tau, x)$. An example of such a surface from this parametric family is given in Figure 4. It was produced using the same parameters as for Figure 3. 


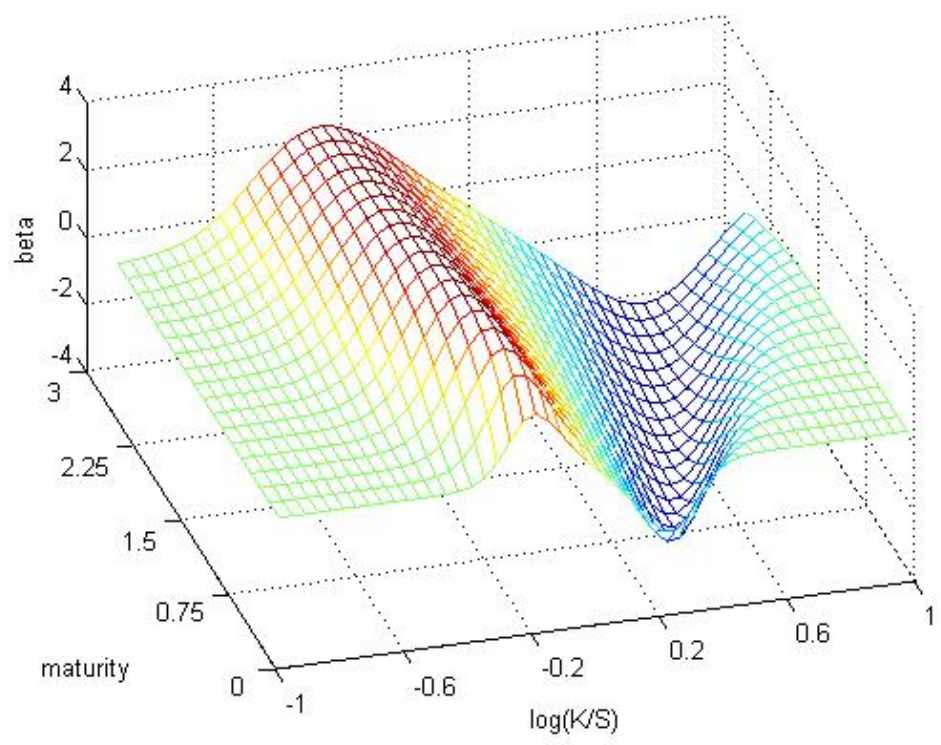

FIGURE 4. Example of a surface from the parametric family identified for the diffusion term in dynamic local volatility models.

It is instructive to compare this plot to the plot of the estimated surface $\hat{\beta}(\tau, x)$ in a Hull-White model where the volatility is modeled as a geometric Brownian motion given in Figure 5. Notice that, despite the fact that these two surfaces clearly differ in scale, they share the same qualitative behavior: for every fixed time to maturity, as functions of $x$ they are close to being odd, and converging to zero at infinity.

We introduced a parametric family for fitting the initial local volatility surface to observed option prices. In addition, we proposed a parametric form for the vol-vol surface $\beta$ which determines the dynamics of the model. The forms of both families are motivated by properties of the popular stochastic volatility models. So despite the fact that general dynamic local volatility models allow for $\hat{a}^{2}$ and $\hat{\beta}$ of much greater generality than those coming from stochastic volatility models, a first approach to dynamic local volatility modeling could use these parameterizations introduced in this section in order to implement the models studied in this paper. We shall report on the results of this strategy in a forthcoming paper.

\section{Hedging With Dynamic Local Volatility}

Notice that everything that has been done so far, was done under a pricing measure $\mathbb{Q}$, and therefore, did not really involve the dynamics under the real world measure $\mathbb{P}$. However, except for some almost sure properties, the analysis of dynamic hedges needs to be done under the real world measure, and we need new assumptions for that. So in this section, we assume that, under the real world measure $\mathbb{P}$ : 


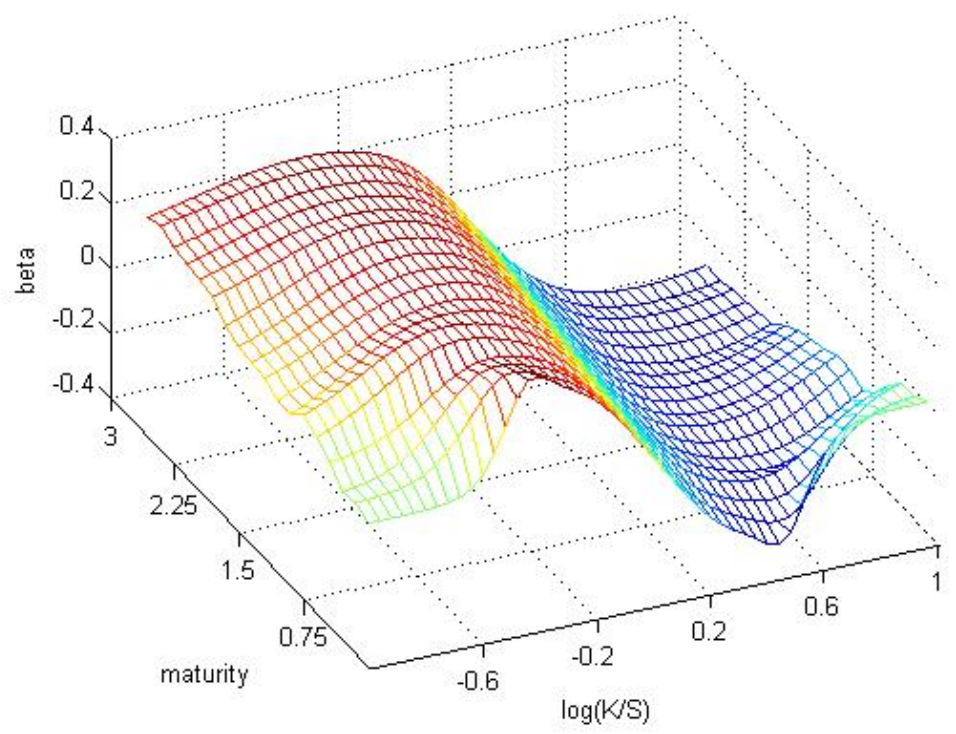

FIGURE 5. Diffusion surface $\hat{\beta}(\tau, x)$ of the local volatility of a Heston model with geometric Brownian motion as volatility.

(1) The stochastic process $B$ which, as a Brownian motion under $\mathbb{Q}$ was driving most of the stochastic differential equations so far, has locally bounded drift. In other words, under $\mathbb{P}$, $B_{t}=A_{t}+W_{t}$. Where $A_{t}=\int_{0}^{t} b_{u} d u$, with the process $\left\|b_{t}(\omega)\right\|$ bounded for all $\omega$ on any finite interval of time, and $W_{t}$ is a Wiener process under $\mathbb{P}$.

(2) The underlying is an $\mathcal{H}^{2}$ semimartingale on any finite time interval, and all the regularity assumptions made in Section 2 hold (this, together with the first assumption, will ensure that the call prices are $\mathcal{H}^{2}$ semimartingales as well).

The dynamic local volatility models introduced in this paper were built under the assumption that the prices of all the European call options were observable and that these options were available for trading. But now that we understand what a no-arbitrage dynamic model means, after stating the elements of such a model, we can consider the more realistic situation, where only finitely many options can be traded liquidly. So, for the purpose of this last section we assume that at each time $t$, the set of liquidly traded options available to the hedger is finite and we denote their values by

$$
\mathcal{C}_{t}=\left\{\hat{C}_{t}^{j} ; j=0,1, \cdots, N\right\}
$$

where

$$
\hat{C}_{t}^{j}=\mathbf{1}_{\left[T_{j}^{1}, T_{j}^{2}\right)}(t) \hat{C}_{t}\left(T_{j}^{2}-t, x_{j}\right), \quad j=0,1, \cdots, N
$$

and where $T_{j}^{1}$ is the time of issuing of the corresponding call option, and $T_{j}^{2}$ is its maturity time, $e^{x_{j}}$ being its strike. The use of the indicator $\mathbf{1}_{[\cdot, \cdot)}$ can be viewed as a justification for the fact that the set of options which we are using as hedging instruments do not change over time. 
This last section is concerned with the hedging of a contingent claim $V$ with time of maturity $T$ and price process $\left(V_{t}\right)_{t \in[0, T]}$ being a $\mathcal{H}^{2}$ semi-martingale, hedging being done by means of a portfolio using the finite set of liquid European call options introduced above.

Definition 1. We call a trading strategy $\delta=\left(\delta_{t}^{0}, \delta_{t}^{1}, \ldots, \delta_{t}^{N}\right)_{t \in[0, T)}$ admissible, if $\delta$ is adapted to $\mathbb{F}$ (which implies predictability, since $\mathbb{F}$ is generated by a Brownian motion), and is square integrable with respect to the hedging instruments, i.e. $\delta \in\left(\mathcal{H}^{2}, \mathcal{C}\right)$. We denote the set of all admissible strategies with maturity $T$ by $\mathcal{A}(T)$.

Admissibility means that $\left(\int_{0}^{t} \delta_{u} \cdot d \mathcal{C}_{u}\right)_{t \in[0, T)}$ is in $\mathcal{H}^{2}$. Recall that the model we consider is possibly incomplete and hedging does not necessarily coincide with replication. Therefore, we need to define the concept of hedging in our setting.

Definition 2. A trading strategy $\bar{\delta}(V)=\left(\bar{\delta}_{t}^{0}(V), \bar{\delta}_{t}^{1}(V), \ldots, \bar{\delta}_{t}^{N}(V)\right)_{t \in[0, T)}$ is a local hedging strategy for the contingent claim $V$ with maturity $T$ if it is admissible, i.e. if $\bar{\delta}(V) \in \mathcal{A}(T)$, and if for almost every $t \in[0, T)$, it holds

$$
\bar{\delta}(V)=\arg \inf _{\delta \in \mathcal{A}(T)} \frac{\bar{\partial}^{+}}{\partial u}\left[\mathbb{E}_{t}^{\mathbb{P}}\left(V_{t+u}-V_{t}-\int_{t}^{t+u} \delta_{v} \cdot d \mathcal{C}_{v}\right)^{2}\right]_{u=0}
$$

where for any real valued function $f$ of a real variable we use the notation $\partial_{x}^{+}$for

$$
\partial_{x}^{+} f(x)=e s s \limsup _{\Delta x \searrow 0} \frac{f(x+\Delta x)-f(x)}{\Delta x} .
$$

Remark 1. It would be more natural to look for the optimal hedging strategy only among those $\delta$ for which

$$
\left(V_{t}-V_{0}-\int_{0}^{t} \delta_{v} \cdot d \mathcal{C}_{v}\right)_{t \in[0, T)}
$$

is a martingale. Then local hedging would become global in the sense of the following definition.

Definition 3. The trading strategy $\tilde{\delta}(V)=\left(\tilde{\delta}_{t}^{0}(V), \tilde{\delta}_{t}^{1}(V), \ldots, \tilde{\delta}_{t}^{N}(V)\right)_{t \in[0, T)}$ is said to be a global hedging strategy for the contingent claim $V$ with maturity $T$ if it is admissible, and, for almost every $t \in[0, T)$, it holds

$$
\tilde{\delta}(V)=\arg \inf _{\delta \in \mathcal{A}(T)} \mathbb{E}^{\mathbb{P}}\left(V_{t}-V_{0}-\int_{0}^{t} \delta_{v} \cdot d \mathcal{C}_{v}\right)^{2}
$$

However, in general, we cannot find an admissible trading strategy $\tilde{\delta}$ attaining the above infimum. Thus, we concentrate on the problem of local hedging and we show how to find for example, the local hedging strategy for a non-liquid call option.

Proposition 6. For any European call option with maturity $T$ and strike $K=e^{x}$, there exists a local hedging strategy $\bar{\delta}_{t}(T, x)$. At every time $t$ it can be computed as a solution of the finite dimensional quadratic optimization problem

$$
\min \left\|H_{t} \delta_{t}-Y_{t}\right\|^{2}
$$


where

$$
Y_{t}=\left(\begin{array}{c}
\hat{\nu}_{t}^{1}(T-t, x) \\
\vdots \\
\hat{\nu}_{t}^{m}(T-t, x)
\end{array}\right), H_{t}=\left(\begin{array}{cccc}
S_{t} \sigma_{t} & \mathbf{1}_{\left[T_{1}^{1}, T_{1}^{2}\right)}(t) \hat{\nu}_{t}^{1}\left(T_{1}^{2}-t, x_{1}\right) & \cdots & \mathbf{1}_{\left[T_{N}^{1}, T_{N}^{2}\right)}(t) \hat{\nu}_{t}^{1}\left(T_{N}^{2}-t, x_{N}\right) \\
0 & \cdots & \cdots & \cdots \\
\vdots & \vdots & \vdots & \vdots \\
0 & \mathbf{1}_{\left[T_{1}^{1}, T_{1}^{2}\right)}(t) \hat{\nu}_{t}^{m}\left(T_{1}^{2}-t, x_{1}\right) & \cdots & \mathbf{1}_{\left[T_{N}^{1}, T_{N}^{2}\right)}(t) \hat{\nu}_{t}^{m}\left(T_{N}^{2}-t, x_{N}\right)
\end{array}\right)
$$

Remarks 1. Recall that $\hat{\nu}_{t}(\tau, x)$ is the diffusion vector of the Itô's decomposition of the semimartingale $\hat{C}_{t}(\tau, x)$ and that it can be computed by solving the system of partial differential equations (48):

2. At each time $t$, the optimization problem is a standard finite dimensional least squares problem which can be solved easily. If $\tilde{H}_{t}$ denotes the matrix consisting of the basis of the columns of $H_{t}$, then the solution $\bar{\delta}_{t}$ is gven by $\left(\tilde{H}_{t}^{T} \tilde{H}_{t}\right)^{-1} \tilde{H}_{t} Y_{t}$.

Proof:

Let the price of non-liquid option at time $t$ be $\hat{C}_{t}(T-t, x)$. For any given admissible trading strategy $\delta$ we define

$$
\begin{aligned}
X_{u} & =\hat{C}_{t+u}(T-(t+u), x)-\hat{C}_{t}(T-t, x)-\sum_{j=0}^{N} \int_{t}^{t+u} \delta_{v}^{j} d \hat{C}_{v}^{j} \\
& =\sum_{i=1}^{m} \int_{t}^{t+u} \eta_{v}^{i} d A_{v}^{i}+\sum_{i=1}^{m} \int_{t}^{t+u} \eta_{v}^{i} d W_{v}^{i}
\end{aligned}
$$

where

$$
\eta_{v}^{1}=\hat{\nu}_{v}^{1}(T-v, x)-\delta_{v}^{0} S_{v} \sigma_{v}-\sum_{j=1}^{N} \mathbf{1}_{\left[T_{j}^{1}, T_{j}^{2}\right)}(v) \delta_{v}^{j} \hat{\nu}_{v}^{1}\left(T_{j}^{2}-v, x_{j}\right)
$$

and

$$
\eta_{v}^{i}=\hat{\nu}_{v}^{i}(T-v, x)-\sum_{j=1}^{N} \mathbf{1}_{\left[T_{j}^{1}, T_{j}^{2}\right)}(v) \delta_{v}^{j} \hat{\nu}_{v}^{i}\left(T_{j}^{2}-v, x_{j}\right), \quad i=2, \ldots, m .
$$

Notice that we obtained the semi-martingale decomposition $X_{u}=\tilde{A}_{u}+\tilde{N}_{u}$, where $\tilde{A}$ is the finite variation component of $X$, and $\tilde{N}$ is the local martingale, and $\tilde{A}_{0}=\tilde{N}_{0}=0$. Notice also that due to the assumptions made at the beginning of this section, and the very definition of the admissible strategies, we have that $\left(X_{t}\right)_{t \in[0, T)} \in \mathcal{H}^{2}$.

$$
X_{u}^{2}=\tilde{A}_{u}^{2}+2 \tilde{A}_{u} \tilde{N}_{u}+\tilde{N}_{u}^{2}
$$

Using $\tilde{A}_{u}=\sum_{i=1}^{m} \int_{t}^{t+u} \eta_{v}^{i} d A_{v}^{i}$, we estimate the first term in (28).

$$
\left(\mathbb{E}^{\mathbb{P}} \tilde{A}_{u}^{2}\right)^{1 / 2} \leq \sum_{i=1}^{m}\left(\mathbb{E}^{\mathbb{P}}\left(\int_{t}^{t+u} \eta_{v}^{i} b_{v}^{i} d v\right)^{2}\right)^{1 / 2} \leq
$$




$$
\sum_{i=1}^{m}\left(\mathbb{E}^{\mathbb{P}} \int_{t}^{t+u}\left|b_{v}^{i}\right| d v \int_{t}^{t+u} \eta_{v}^{i^{2}}\left|b_{v}^{i}\right| d v\right)^{1 / 2} \leq c \cdot \sqrt{u} \cdot \sum_{i=1}^{m}\left(\mathbb{E}^{\mathbb{P}} \int_{t}^{t+u} \eta_{v}^{i^{2}} d v\right)^{1 / 2}
$$

The last estimate implies that $\tilde{A}_{u}^{2} / u$ converges to zero in $L^{1}$, as $u \rightarrow 0$. Clearly, it also does so in almost sure sense. Thus

$$
\lim _{u \rightarrow 0} \frac{\mathbb{E}_{t}^{\mathbb{P}} \tilde{A}_{u}^{2}}{u}=0
$$

We estimate the second term by

$$
\mathbb{E}^{\mathbb{P}} \tilde{A}_{u} \tilde{N}_{u} \leq\left(\mathbb{E}^{\mathbb{P}} \tilde{A}_{u}^{2}\right)^{1 / 2}\left(\mathbb{E}^{\mathbb{P}} \tilde{N}_{u}^{2}\right)^{1 / 2}=\left(\mathbb{E}^{\mathbb{P}} \tilde{A}_{u}^{2}\right)^{1 / 2}\left(\mathbb{E}^{\mathbb{P}} \sum_{i=1}^{m} \int_{t}^{t+u} \eta_{v}^{i^{2}} d v\right)^{1 / 2}
$$

Obviously, $\int_{0}^{t} \mathbb{E}^{\mathbb{P}} \eta_{v}^{i} d v$ is differentiable in almost every $t$, so if we consider any such $t$, then, as $u \rightarrow 0$

$$
\frac{\mathbb{E}^{\mathbb{P}} \tilde{A}_{u} \tilde{N}_{u}}{u} \leq\left(\mathbb{E}^{\mathbb{P}} \frac{\tilde{A}_{u}^{2}}{u}\right)^{1 / 2}\left(\frac{\sum_{i=1}^{m} \int_{t}^{t+u} \mathbb{E}^{\mathbb{P}} \eta_{v}^{i} d v}{u}\right)^{1 / 2} \rightarrow 0
$$

which implies, as before, that

$$
\lim _{u \rightarrow 0} \frac{\mathbb{E}_{t}^{\mathbb{P}} \tilde{A}_{u} \tilde{N}_{u}}{u}=0
$$

Finally, the last term in (28) gives:

$$
\limsup _{u \rightarrow 0} \frac{\mathbb{E}_{t}^{\mathbb{P}} \tilde{N}_{u}^{2}}{u}=\limsup _{u \rightarrow 0} \frac{1}{u} \mathbb{E}^{\mathbb{P}} \sum_{i=1}^{m} \int_{t}^{t+u} \eta_{v}^{i^{2}} d v
$$

due to the definition of $\eta$

$$
\begin{gathered}
\sum_{i=1}^{m} \int_{t}^{t+u} \eta_{v}^{i^{2}} d v=\int_{t}^{t+u}\left(\hat{\nu}_{v}^{1}(T-v, x)-\delta_{v}^{0} S_{v} \sigma_{v}-\sum_{j=1}^{N} I_{\left[T_{j}^{1}, T_{j}^{2}\right)}(v) \delta_{v}^{j} \hat{\nu}_{v}^{1}\left(T_{j}^{2}-v, x_{j}\right)\right)^{2}+ \\
\sum_{i=2}^{m}\left(\hat{\nu}_{v}^{i}(T-v, x)-\sum_{j=1}^{N} I_{\left[T_{j}^{1}, T_{j}^{2}\right)}(v) \delta_{v}^{j} \hat{\nu}_{v}^{i}\left(T_{j}^{2}-v, x_{j}\right)\right)^{2} d v
\end{gathered}
$$

Notice that, for fixed $(\omega, v)$, the integrand is minimized by $\bar{\delta}_{v}$ specified in the proposition. Clearly, $\bar{\delta}$ is an adapted process. Also, $\left(H_{t} \bar{\delta}_{t}\right)_{t \in[0, T)}$ is a square integrable process, since, for every $t$, it is bounded by $\left\|Y_{t}\right\|$, as projection of $Y_{t}$. It follows that $\int_{0}^{t} \delta_{u} \cdot d \mathcal{C}_{u}$ is in $\mathcal{H}^{2}$, and therefore $\bar{\delta}$ is admissible.

Now, denote $\eta$, corresponding to $\bar{\delta}$, by $\bar{\eta}$. It is clear that for any other admissible strategy $\delta$, we have

$$
\limsup _{u \rightarrow 0} \frac{1}{u} \mathbb{E}_{t}^{\mathbb{P}} \sum_{i=1}^{m} \int_{t}^{t+u} \bar{\eta}_{v}^{i 2} d v \leq \limsup _{u \rightarrow 0} \frac{1}{u} \mathbb{E}_{t}^{\mathbb{P}} \sum_{i=1}^{m} \int_{t}^{t+u} \eta_{v}^{i^{2}} d v
$$

almost surely, which completes the proof. 
Remark that for any portfolio of call options (i.e. a finite linear combination $\sum_{k=1}^{M} p_{k} \hat{C}_{t}\left(\hat{T}^{k}\right.$ $\left.t, x_{k}\right)$ ), the hedging strategy is clearly given by Proposition 6 , with the same $H_{t}$ and

$$
Y_{t}=\sum_{k=1}^{M} p_{k} Y_{t}^{k}
$$

where

$$
Y_{t}^{k}=\left(\begin{array}{c}
\hat{\nu}^{1}\left(\hat{T}^{k}-t, \hat{x}^{k}\right) \\
\ldots \\
\hat{\nu}^{m}\left(\hat{T}^{k}-t, \hat{x}^{k}\right)
\end{array}\right)
$$

It is also easy to see that any measurable payoff $F\left(S_{T}\right)$ is in the closure (for example, in $L^{1}$ sense) of the linear combinations of $\left(S_{T}-K\right)^{+}{ }_{K \geq 0}$. Thus, under some integrability conditions, we can find the hedge of contingent claim with payoff $F\left(S_{T}\right)$ as a limit of hedging strategies for linear combinations of vanilla options. And doing this will only change the projection vector $Y$, which now will be a limit of the vectors corresponding to linear combinations of vanilla options.

Proposition 6 gives a local hedging strategy but it does not address how well or how poorly such a strategy performs in global (replication) sense. In particular, what can we say about the terminal value of the hedging portfolio? For a partial answer to this difficult question, we assume for example that the contingent claim $V$ is a linear combination of vanilla options with the same maturity, then we can use the following inequality which is a straightforward application of Theorem 2 p. 252 in Protter's book [28].

$$
\begin{aligned}
\mathbb{E}^{\mathbb{P}}\left[\sup _{t \in[0, T]}\right. & \left.\left(V_{t}-V_{0}-\int_{0}^{t} \delta_{v} \cdot d \mathcal{C}_{v}\right)^{2}\right] \\
\leq & c \cdot\left(\left(\mathbb{E}^{\mathbb{P}} \int_{0}^{T}\left(\left(H_{t} \delta_{t}-Y_{t}\right) \cdot b_{t}\right)^{2} d t\right)^{1 / 2}+\left(\mathbb{E}^{\mathbb{P}} \int_{0}^{T}\left\|H_{t} \delta_{t}-Y_{t}\right\|^{2} d t\right)^{1 / 2}\right)^{2} \\
\leq & c \cdot \mathbb{E}^{\mathbb{P}} \int_{0}^{T}\left\|H_{t} \delta_{t}-Y_{t}\right\|^{2} d t
\end{aligned}
$$

Where the last constant depends only on the $\operatorname{ess} \sup _{\omega, t \in[0, T]}\left\|b_{t}(\omega)\right\|$ (recall that $b$ is the drift of $B$ under $\mathbb{P}$ ). Thus, we can see that solving the minimization problem in Proposition 6 at every time $t$, will give us an idea of how low the P\&L may fall during the life of the portfolio.

\section{Conclusion}

In this paper, we propose a new market model for the equity markets. Its originality is to be based on the prescription of stochastic dynamics for the local volatility surface. We argue that the local volatility surface is a better description of the state of the market than the call option prices surface and the implied volatility surface. The main thrust of the paper is a characterization of the absence of arbitrage by means of a drift condition and a spot consistency condition in line with the original Heath-Jarrow-Morton absence of arbitrage condition. As a by-product of our results, we characterize the Markov spot models as those with local volatility bounded variations dynamics, shedding some new light on the original works of Dupire and Derman and Kani who introduced the concept of local volatility surface in the static context. We also provide a new expression for the local volatility 
surfaces of stochastic volatility models, we introduce a parametric family of local volatility surfaces, and we demonstrate that it is well suited to the analysis of actual financial data. Finally, we consider the problem of hedging in the framework of market models for the local volatility surface.

\section{APPENDiX A}

\section{Proof of Lemma 1.}

Fix some $\bar{t}$ and $\bar{\tau}, \bar{x}$, and consider only $t \in[0, \bar{t}], \tau \in[0, \bar{\tau}]$. In the following, we often omit the subscript $t$ in expressions that are dynamic due to the fact that most of the time $t$ is actually fixed.

First consider the explicit Euler finite difference scheme for (7). Choose some small $\tau, h>0$, and define $\Lambda(\tau, h):=\left\{(\tau n, h k) \mid n \in \mathbb{N}^{*}, k=\mathbb{Z}\right\}$ for the two dimensional lattice with $\operatorname{mesh}(\Lambda)=$ $\max (\tau, h)$ where $\tau$ and $h$ are always chosen in such a way that the lattice contains $(\bar{\tau}, \bar{x})$.

Let $\eta_{h}$ and $\eta_{-h}$ denote the right and left shift operators (along the $x$ axis) respectively, and

$$
\begin{gathered}
d_{h}=\frac{1}{h} \eta_{h}, \quad d_{-h}=\frac{1}{h} \eta_{-h} \\
D_{h}=\frac{1}{2}\left(d_{-h} d_{h}-d_{h}\right) \\
L_{h}=\hat{a}^{2} D_{h}
\end{gathered}
$$

be the discrete analogues of $\partial_{x}, D_{x}$ and $L_{x}$ respectively. Whenever we have a function $f$ defined on the lattice, we use the notation $f_{n, k}$ for $f(\tau n, h k)$. We call $\bar{C}(\Lambda)$ the solution of the explicit finite difference scheme for (7), i.e.

$$
\left\{\begin{array}{l}
\bar{C}_{n+1, k}=\bar{C}_{n, k}+\tau L_{h} \bar{C}_{n, k}, \quad n \in \mathbb{N}^{*}, k=\mathbb{Z} \\
\bar{C}_{0, k}=F_{\varepsilon}\left(S-e^{h k}\right), \quad k=\mathbb{Z} .
\end{array}\right.
$$

From now on, we consider only those $(\tau, h)$ for which the ratio $\tau / h^{2}$ is small enough, and therefore, the above scheme is stable. We fix $\Lambda$ and $(n, k)$. Since $\Lambda$ does not depend upon $t, \bar{C}_{n, k}^{\varepsilon}$ is an adapted process in $t \in[0, \bar{t}]$. It is clearly an Itô process, as a polynomial of Itô processes. We write its decomposition as

$$
d\left(\bar{C}_{n, k}(\Lambda)\right)_{t}=\bar{\mu}_{n, k, t}(\Lambda) d t+\bar{\nu}_{n, k, t}(\Lambda) \cdot d B_{t} .
$$

Using the Ito's formula and varying $(n, k)$, we conclude that the elements of this decomposition satisfy the following equations, a.s. for every $t \in[0, \bar{t}]$

$$
\left\{\begin{array}{l}
\bar{\nu}_{n+1, k}^{i}=\bar{\nu}_{n, k}^{i}+\frac{\tau}{h^{2}} L_{h} \bar{\nu}_{n, k}^{i}+\frac{\tau}{h^{2}} \hat{\beta}_{n, k}^{i} L_{h} \bar{C}_{n, k}, \quad i=1, \ldots, m \\
\bar{\nu}_{0, k}^{1}=S \sigma F_{\varepsilon}^{\prime}(S-\exp (h k)) \\
\bar{\nu}_{0, k}^{i}=0, \quad i=2, \ldots, m
\end{array}\right.
$$

and

$$
\left\{\begin{array}{l}
\bar{\mu}_{n+1, k}=\bar{\mu}_{n, k}+\frac{\tau}{h^{2}} L_{h} \bar{\mu}_{n, k}+\frac{\tau}{h^{2}} \hat{\alpha}_{n, k} L_{h} \bar{C}_{n, k}+\frac{\tau}{h^{2}} \hat{\beta}_{n, k} \cdot L_{h} \bar{\nu}_{n, k} \\
\bar{\mu}_{0, k}=\frac{1}{2} S_{t}^{2} \sigma_{t}^{2} F_{\varepsilon}^{\prime \prime}(S-\exp (h k)) .
\end{array}\right.
$$


Now let us consider (31). Using the smoothness of the initial condition and of the coefficient $\hat{a}^{2}(T, x)$, and following the lines of [20] p. 230-233, we derive the following estimate

$$
\text { || }\left|D_{h} \bar{C} \|\right| \leq c \cdot e^{c \cdot\left|\left\|\partial_{x}^{2} \hat{a}^{2}\right\|\right|}
$$

where $|\|\cdot\||$ is the uniform norm over the strip $[0, \bar{T}] \times \mathbb{R}$. As usual $c$ denotes a constant which may vary from line to line, but which does not dependent upon $\Lambda, t$ and $\omega$. In a similar manner we obtain

$$
\left.\left|\left\|d_{h}^{k} D_{h} \bar{C}\right\|\right| \leq c \cdot e^{c \cdot \sum_{i=1}^{2}\left|\left\|\partial_{x}^{i} \hat{a}^{2}\right\|\right|} \cdot\left(1+\left(\sum_{i=2}^{k+2}\left|\left\|\partial_{x}^{i} \hat{a}^{2}\right\|\right|\right)\right)^{k}\right) \quad k=0,1,2,3
$$

The last estimate, together with Arzela-Asccoli's theorem (see, for example, [20] p. 230-233), implies that $\bar{C}$ converges to $\hat{C}^{\varepsilon}$, in the norm $\|$.$\| \| , along an increasing sequence of lattices \Lambda_{N}$ (i.e. $\Lambda_{N} \subset$ $\Lambda_{N+1}$ ), whose meshes decrease to 0 . Moreover, the convergence holds as well for the first two derivatives of $\bar{C}$ and $\hat{C}^{\varepsilon}$ with respect to $x$.

We now consider (32). Without any loss of generality, we fix $i=1$, and introduce $\check{\nu}$ as a solution of the following numerical scheme

$$
\left\{\begin{array}{l}
\check{\nu}_{n+1, k}=\check{\nu}_{n, k}+\tau L_{h} \check{\nu}_{n, k}+\tau \hat{\beta}_{n, k}^{1} L_{h} \hat{C}_{n, k}^{\varepsilon} \\
\check{\nu}_{0, k}=S \sigma F_{\varepsilon}^{\prime}(S-\exp (h k)) .
\end{array}\right.
$$

Notice that the difference $\bar{\nu}^{1}-\check{\nu}$ satisfies a similar scheme with initial condition being zero, and having $\hat{\beta}^{1}\left(L_{h} \bar{C}^{\varepsilon}-L_{h} \hat{C}^{\varepsilon}\right)$ as the inhomogeneous part. Therefore, we have the estimate

$$
\left|\left\|\bar{\nu}^{1}-\check{\nu}\right\|\right|_{1} \leq\left|\left\|\hat{\beta}^{1}\right\|\right|_{1} \cdot\left|\left\|\hat{a}^{2}\right\|\right| \cdot\left|\left\|D_{h} \hat{C}^{\varepsilon}-D_{h} \bar{C}^{\varepsilon}\right\|\right|,
$$

with $|\|f\||_{j}=\left|\left\|f \times e^{-j|x|}\right\|\right|$. The right hand side of the last inequality, as it was shown above, goes to zero when $\operatorname{mesh}\left(\Lambda_{N}\right) \searrow 0$. Also, we notice that (35) is an explicit Euler scheme for the partial differential equation:

$$
\left\{\begin{array}{l}
\partial_{\tau} \hat{\nu}^{\varepsilon, 1}=L_{x} \hat{\nu}^{\varepsilon, 1}+\hat{\beta}^{1} L_{x} \hat{C}^{\varepsilon}, \quad \tau>0, x \in \mathbb{R} \\
\hat{\nu}^{\varepsilon, 1}(0, x)=S \sigma F_{\varepsilon}^{\prime}\left(S-e^{x}\right)
\end{array}\right.
$$

The explicit Euler scheme applied to the linear partial differential equations (37), is consistent in the space of continuous functions with any of the norms $\mid\|\cdot\| \|_{j}$ (see, for example, [27], or [29]). And since we know from (34) that $D_{x} \hat{C}^{\varepsilon}$ is bounded in the strip $[0, \bar{\tau}] \times \mathbb{R}$, and since the initial condition of (37) is clearly infinitely smooth with all derivatives bounded, the stability of the scheme follows. Moreover, from (43), we see that $D_{x} \hat{C}^{\varepsilon}(\tau, x)$ is continuous and converges to zero as $|x| \rightarrow \infty$, uniformly in $\tau \in[0, \bar{\tau}]$. This implies uniform continuity for $\sup _{x \in \mathbb{R}}\left|e^{-|x|} \hat{\beta}^{1} L_{x} \hat{C}^{\varepsilon}\right|$ as a function of $\tau$. Consistency and stability of the numerical scheme, together with uniform continuity of the norm of inhomogeneous part, yield the convergence of $\check{\nu}$ to $\hat{\nu}^{\varepsilon, 1}$, as $N \rightarrow \infty$ (see [27], or [29]). Together with (36), it implies the convergence of $\bar{\nu}^{1}$ to $\hat{\nu}^{\varepsilon, 1}$, in |\|. $\|\left.\right|_{1}$.

Now, we need estimates on the derivatives of $\bar{\nu}^{1}$. Applying the method used to derive (34), to equation (32), we obtain 


$$
\left|\left\|d_{ \pm h}^{k} \bar{\nu}^{1}\right\|\right|_{1} \leq c \cdot e^{c \cdot \sum_{i=1}^{2}\left|\left\|\partial_{x}^{i} \hat{a}^{2}\right\|\right|} \cdot\left(1+\left(\sum_{i=1}^{k}\left|\left\|\partial_{x}^{i} \hat{\beta}\right\|\right|_{1}\right)\left(1+\left(\sum_{i=2}^{2+k}\left|\left\|\partial_{x}^{i} \hat{a}^{2}\right\|\right|\right)^{k}\right)\right)
$$

for $k=0,1,2,3$. Again, we use Arzela-Asccoli's theorem to conclude that $D_{h} \bar{\nu}^{1} \rightarrow D \hat{\nu}^{\varepsilon, 1}$ in $\mid\|\cdot\| \|_{1}$, along some subsequence of $\left\{\Lambda_{N}\right\}$ (and without any loss of generality, we can assume that it is $\left\{\Lambda_{N}\right\}$ itself). The same holds for $\bar{\nu}^{i}$, with $i>1$. Now introduce the solution $\check{\mu}$ of

$$
\left\{\begin{array}{l}
\check{\mu}_{n+1, k}=\check{\mu}_{n, k}+\tau L_{h} \check{\mu}_{n, k}+\tau \hat{\alpha}_{n, k} L_{h} \hat{C}_{n, k}+\tau \hat{\beta}_{n, k} \cdot L_{h} \hat{\nu}_{n, k}^{\varepsilon} \\
\check{\mu}_{0, k}=\frac{1}{2} S^{2} \sigma^{2} F_{\varepsilon}^{\prime \prime}\left(S-e^{h k}\right)
\end{array}\right.
$$

which is the explicit Euler scheme for

$$
\left\{\begin{array}{c}
\partial_{\tau} \hat{\mu}^{\varepsilon}=L_{x} \hat{\mu}^{\varepsilon}+\hat{\alpha} L_{x} \hat{C}^{\varepsilon}+\hat{\beta} \cdot L_{x} \hat{\nu}^{\varepsilon}, \quad \tau>0, x \in \mathbb{R} \\
\hat{\mu}^{\varepsilon}(0, x)=\frac{1}{2} S^{2} \sigma^{2} F_{\varepsilon}^{\prime \prime}\left(S-e^{x}\right) .
\end{array}\right.
$$

Considering the numerical scheme for $\bar{\mu}-\check{\mu}$, as it was done for $\bar{\nu}^{1}-\check{\nu}$, we conclude that $|\|\bar{\mu}-\check{\mu}\||_{2} \rightarrow$ 0 along $\left\{\Lambda_{N}\right\}$. Notice that we have also shown that

$$
\left.\left\|D_{x} \hat{C}^{\varepsilon}\right\|\right|_{1}<\infty, \quad \sum_{i=1}^{m} \quad \mid\left\|D_{x} \hat{\nu}^{\varepsilon, i}\right\|_{1}<\infty
$$

therefore

$$
\text { | }\left.\left\|\hat{\alpha} L_{x} \hat{C}^{\varepsilon}\right\|\right|_{2}<\infty, \quad\left\|\hat{\beta} \cdot L_{x} \hat{\nu}^{\varepsilon}\right\|_{2}<\infty
$$

This implies stability for (39). As before, we deduce that the norm of the inhomogeneous part,

$$
\sup _{x \in \mathbb{R}}\left|e^{-2|x|} \hat{\alpha} L_{x} \hat{C}^{\varepsilon}+e^{-2|x|} \hat{\beta} \cdot L_{x} \hat{\nu}^{\varepsilon}\right|,
$$

is uniformly continuous in $\tau$. And it means that $\check{\mu}$ converges to $\hat{\mu}^{\varepsilon}$. Therefore, $\bar{\mu} \rightarrow \hat{\mu}^{\varepsilon}$ along $\Lambda_{N}$. Moreover, as on side, we have the estimate

$$
|\|\bar{\mu}\||_{2} \leq c+|\|\hat{\alpha}\||_{2} \cdot\left|\left\|\hat{a}^{2}\right\|\right| \cdot\left|\left\|D_{h} \bar{C}\right\|\right|+\left|\left\|\hat{\beta}||_{1} \cdot\left|\left\|\hat{a}^{2}\right\|\right| \cdot\left|\left\|\sum_{i=1}^{m} D_{h} \bar{\nu}^{i}\right\|\right| .\right.\right.
$$

Applying (34) and (38), we continue

$\|\bar{\mu}\|_{2} \leq c \cdot\left(1+|\|\hat{\alpha}\||_{2} \cdot\left|\left\|\hat{a}^{2}\right\|\right| \cdot e^{c \cdot \sum_{i=1}^{2}\left|\left\|\partial_{x}^{i} \hat{a}^{2}\right\|\right|} \cdot\left(1+\left(\sum_{i=1}^{2}\left|\left\|\partial_{x}^{i} \hat{\beta}\right\|\right|_{1}\right)\left(1+\left(\sum_{i=2}^{4}\left|\left\|\partial_{x}^{i} \hat{a}^{2}\right\|\right|\right)^{2}\right)\right)\right)$

The same estimate holds for $\hat{\mu}^{\varepsilon}$. Using the notation introduced in Section 2, we have that, for any $t \in[0, \bar{t}]$, both $\bar{\mu}_{t}$ and $\hat{\mu}_{t}^{\varepsilon}$ are bounded by

$$
c \cdot\left(1+p_{2}^{5}(t) \cdot e^{c \cdot \lambda_{1}(t)}\right)
$$


Similarly, from (38) we deduce that $\bar{\nu}_{t}^{i}$ and $\hat{\nu}_{t}^{\varepsilon, i}$ are bounded from above by $c \cdot e^{c \cdot \lambda_{1}(t)}$ for any $i=$ $1, \ldots, m$. Thus for any $t \in[0, \bar{t}]$, we have when $N \rightarrow \infty$ that

$$
\begin{aligned}
\bar{C}_{t}\left(\bar{\tau}, \bar{x}, \Lambda_{N}\right) & \rightarrow \hat{C}_{t}^{\varepsilon}(\bar{\tau}, \bar{x}) \\
\bar{\nu}_{t}\left(\bar{\tau}, \bar{x}, \Lambda_{N}\right) & \rightarrow \hat{\nu}_{t}^{\varepsilon}(\bar{\tau}, \bar{x}) \\
\bar{\mu}_{t}\left(\bar{\tau}, \bar{x}, \Lambda_{N}\right) & \rightarrow \hat{\mu}_{t}^{\varepsilon}(\bar{\tau}, \bar{x})
\end{aligned}
$$

Using the fact that

$$
\bar{C}_{t}\left(\bar{\tau}, \bar{x}, \Lambda_{N}\right)=\bar{C}_{0}\left(\bar{\tau}, \bar{x}, \Lambda_{N}\right)+\int_{0}^{t} \bar{\mu}_{u}\left(\bar{\tau}, \bar{x}, \Lambda_{N}\right) d u+\int_{0}^{t} \bar{\nu}_{u}\left(\bar{\tau}, \bar{x}, \Lambda_{N}\right) \cdot d B_{u}
$$

(notice that $\hat{\mu}^{\varepsilon}(\bar{\tau}, \bar{x})$ and $\hat{\nu}^{\varepsilon}(\bar{\tau}, \bar{x})$ are predictable by construction) the bounds derived above and the dominated convergence theorem, we deduce that, for any $t \in[0, \bar{t}]$

$$
\lim _{N \rightarrow \infty} \int_{0}^{t} \bar{\mu}_{u}\left(\bar{\tau}, \bar{x}, \Lambda_{N}\right) d u=\int_{0}^{t} \hat{\mu}_{u}^{\varepsilon}(\bar{\tau}, \bar{x}) d u \quad \text { a.s. }
$$

This implies that $\int_{0}^{t} \bar{\nu}_{u}\left(\bar{\tau}, \bar{x}, \Lambda_{N}\right) \cdot d B_{u}$ a.s. has a limit, as $N \rightarrow \infty$. On the other hand, again from dominated convergence, we have that for any $t \in[0, \bar{t}]$

$$
\lim _{N \rightarrow \infty} \mathbb{E} \int_{0}^{t}\left\|\bar{\nu}_{u}\left(\bar{\tau}, \bar{x}, \Lambda_{N}\right)-\hat{\nu}_{u}^{\varepsilon}(\bar{\tau}, \bar{x})\right\|^{2} d u=0
$$

almost surely, which yields

$$
\int_{0}^{t} \bar{\nu}_{u}\left(\bar{\tau}, \bar{x}, \Lambda_{N}\right) \cdot d B_{u} \rightarrow \int_{0}^{t} \hat{\nu}_{u}^{\varepsilon}(\bar{\tau}, \bar{x}) \cdot d B_{u}
$$

in probability. But, since we also know it converges almost surely, the limits should be the same, and we conclude that

$$
\hat{C}_{t}^{\varepsilon}(\bar{\tau}, \bar{x})=\hat{C}_{0}^{\varepsilon}(\bar{\tau}, \bar{x})+\int_{0}^{t} \hat{\mu}_{u}^{\varepsilon}(\bar{\tau}, \bar{x}) d u+\int_{0}^{t} \hat{\nu}_{u}^{\varepsilon}(\bar{\tau}, \bar{x}) \cdot d B_{u}
$$

This decomposition holds true for any choice of $\bar{t}, \bar{\tau}, \bar{x}$. In addition, almost surely, $\hat{\mu}_{t}^{\varepsilon}$ and $\hat{\nu}_{t}^{\varepsilon}$ have values in $C^{1,2}\left(\mathbb{R}_{+} \times \mathbb{R}\right)$ by construction. This completes the proof.

\section{Proof of Lemma 2}

As before, we restrain $(t, \tau)$ to a fixed compact set $[0, \bar{t}] \times[0, \bar{\tau}]$, and for the sake of notation the constants $c$ can depend upon $(\bar{t}, \bar{\tau})$.

We fix $\omega, t$ and we let $\varepsilon \searrow 0$. From dominated convergence (recall the choice of the approximation functions $\left.F_{\varepsilon}\right)$ it is clear that $\hat{C}^{\varepsilon}(\tau, x) \rightarrow \hat{C}(\tau, x)$ for every $\tau>0, x \in \mathbb{R}$. Recall that, Feynman-Kac formula implies that

$$
D_{x} \hat{C}^{\varepsilon}(\tau, x)=\int_{\mathbb{R}} D_{x} \hat{p}(\tau, x ; 0, y) F_{\varepsilon}\left(S-e^{y}\right) d y
$$

where $\hat{p}(\tau, x ; v, y), \tau<v$, is the fundamental solution of the partial differential equation (5). Since (5) is uniformly parabolic, we can use well known estimates on the fundamental solutions (see for example, [19]), allowing us to apply Fubini's theorem and interchange the integration and differentiation. Since

$$
\left|D_{x} \hat{p}(\tau, x ; 0, y) F_{\varepsilon}\left(S-e^{y}\right)\right| \leq(S+\varepsilon)\left|D_{x} \hat{p}(\tau, x ; 0, y)\right|
$$


and since the last expression is integrable in $y$, we can apply the dominated convergence theorem, and conclude that

$$
D_{x} \hat{C}^{\varepsilon}(\tau, x) \rightarrow \int_{\mathbb{R}} D_{x} \hat{p}(\tau, x ; 0, y) F\left(S-e^{y}\right) d y=D_{x} \hat{C}(\tau, x)
$$

as $\varepsilon \rightarrow 0$. Next we introduce the fundamental solution $\hat{q}(v, y ; \tau, x)$ of

$$
\partial_{v} u(v, y)=-\frac{1}{2} \hat{a}_{t}^{2}(v, y)\left(\partial_{y y}^{2} u(v, y)-\partial_{y} u(v, y)\right)
$$

which is in a sense, dual to (5). The exact relationship between (5) and (42), as well as $\hat{p}$ and $\hat{q}$, is discussed in [12]. We will only use the fact that $D_{x} \hat{p}(\tau, x ; v, y)=e^{x-y} D_{y} \hat{q}(v, y ; \tau, x)$. Let us now derive several useful equalities. Starting from

$$
D_{x} \hat{C}^{\varepsilon}(\tau, x)=\int_{\mathbb{R}} e^{x-y} D_{y} \hat{q}(0, y ; \tau, x) F_{\varepsilon}\left(S-e^{y}\right) d y
$$

and integrating by parts we get

$$
D_{x} \hat{C}^{\varepsilon}(\tau, x)=\int_{\mathbb{R}} e^{x} \hat{q}(0, y ; \tau, x) D_{y}^{*}\left(e^{-y} F_{\varepsilon}\left(S-e^{y}\right)\right) d y
$$

where $D_{y}^{*}=\frac{1}{2}\left(\partial_{y y}^{2}+\partial_{y}\right)$. Computing the derivatives, we obtain

$$
D_{x} \hat{C}^{\varepsilon}(\tau, x)=\frac{1}{2} \int_{\mathbb{R}} \hat{q}(0, y ; \tau, x) e^{x+y} F_{\varepsilon}^{\prime \prime}\left(S-e^{y}\right) d y .
$$

Similarly

$$
\begin{aligned}
D_{x} \hat{C}(\tau, x) & =\int_{\mathbb{R}} D_{x} \hat{p}(\tau, x ; 0, y) F_{0}\left(S-e^{y}\right) d y \\
& =\int_{\mathbb{R}} e^{x-y} D_{y} \hat{q}(0, y ; \tau, x)\left(S-e^{y}\right)^{+} d y \\
& =\frac{1}{2} e^{x} \hat{q}(0, \log S ; \tau, x) .
\end{aligned}
$$

Now consider $\hat{\nu}^{\varepsilon, i}(\tau, x)$ for $i=2, \ldots, m$. The Feynman-Kac formula gives

$$
\hat{\nu}^{\varepsilon, i}(\tau, x)=\int_{\mathbb{R}} \int_{0}^{T} \hat{p}(\tau, x ; u, y) \hat{\beta}^{i}(u, y) \hat{a}^{2}(u, y) D_{y} \hat{C}^{\varepsilon}(u, y) d u d y
$$

Recall that $\hat{\beta}^{i}(u, y)$ is $O\left(e^{|y|}\right)$, and that $\hat{a}^{2}(u, y)$ is bounded, so using (43), (44), and the estimates on the increments of the derivatives of a fundamental solution given on p.255 of [19], we obtain the 
following estimate

$$
\begin{aligned}
& \left|\left(\partial_{y}-1\right)^{k}\left(D_{y} \hat{C}^{\varepsilon}(u, y)-D_{y} \hat{C}(u, y)\right)\right| \\
& =\frac{1}{2} e^{y}\left|\int_{\mathbb{R}} \partial_{y}^{k} \hat{q}(0, z ; u, y) e^{z} F_{\varepsilon}^{\prime \prime}\left(S-e^{z}\right) d z-\partial_{y}^{k} \hat{q}(0, \log S ; u, y)\right| \\
& \leq \frac{1}{2} e^{y} \int_{-1}^{1 \wedge \frac{S}{\varepsilon}} \varphi(z)\left|\partial_{y}^{k} \hat{q}(0, \log (S-\varepsilon z) ; u, y)-\partial_{y}^{k} \hat{q}(0, \log S ; u, y)\right| d z \\
& \leq c(t, \omega) \cdot u^{-\frac{3 / 2+k}{2}} \cdot e^{y} \cdot \\
& \quad \int_{-1}^{1 \wedge \frac{S}{\varepsilon}} \varphi(z)\left(\log \left(1-\frac{\varepsilon}{S} z\right)\right)^{1 / 2}\left(\exp \left[-c \frac{(\log (S-\varepsilon z)-y)^{2}}{u}\right]+\exp \left[-c \frac{(\log S-y)^{2}}{u}\right]\right) d z
\end{aligned}
$$

for $k=0,1$ (recall that the function $\varphi$ was introduced at the beginning of Section 2). For the rest of the proof, the positive constants $c$ do not depend on $\omega, t, \varepsilon$.

Due to the regularity assumptions, $c(t, \omega)$ in the expression above is bounded by $c \cdot e^{c \cdot \lambda_{1}(t)}$, where the process $\lambda_{1}$ was introduced in Section 2. Using (47) and estimates on fundamental solutions, we get

$$
\begin{aligned}
\left|\int_{0}^{\tau} \int_{\mathbb{R}} \hat{p}(\tau, x ; u, y) \hat{a}^{2}(u, y) \hat{\beta}^{i}(u, y)\left(D_{y} \hat{C}^{\varepsilon}(u, y)-D_{y} \hat{C}(u, y)\right) d y d u\right| \\
\leq c \cdot \lambda_{2}^{2} \cdot e^{c \cdot \lambda_{1}} \cdot \int_{-1}^{1 \wedge \frac{S}{\varepsilon}} \varphi(z)\left(\log \left(1-\frac{\varepsilon}{S} z\right)\right)^{1 / 2} \cdot \\
\quad\left(\int_{0}^{\tau} \int_{\mathbb{R}} \frac{1}{(\tau-u)^{1 / 2}} \exp \left(-c \frac{(x-y)^{2}}{\tau-u}\right) e^{2|y|} \frac{1}{u^{3 / 4}} \exp \left(-c \frac{(\log (S-\varepsilon z)-y)^{2}}{u}\right) d y d u\right. \\
\left.\quad+\int_{0}^{\tau} \int_{\mathbb{R}} \frac{1}{(\tau-u)^{1 / 2}} \exp \left(-c \frac{(x-y)^{2}}{\tau-u}\right) e^{2|y|} \frac{1}{u^{3 / 4}} \exp \left(-c \frac{(\log S-y)^{2}}{u}\right) d y d u\right) d z .
\end{aligned}
$$

Now, we fix $\delta>0$ small enough so that $e^{2|y|} \leq c(\delta) e^{\delta y^{2}}$ and

$$
-c \frac{(x-y)^{2}}{\tau-u}+\delta(x-y)^{2} \leq-c_{1} \frac{(x-y)^{2}}{\tau-u}
$$

for some $c_{1}>0$. Such a choice is possible because $\tau-u$ changes on a compact set. This will take care of the $e^{2|y|}$ term inside the integrands. Continuing with the first integral we get

$$
\begin{aligned}
& \int_{0}^{\tau} \int_{\mathbb{R}} \frac{1}{(\tau-u)^{1 / 2}} \exp \left[-c \frac{(x-y)^{2}}{\tau-u}\right] e^{2|y|} \frac{1}{u^{3 / 4}} \exp \left[-c \frac{(\log (S-\varepsilon z)-y)^{2}}{u}\right] d y d u \\
& \quad \leq c(\delta) \cdot e^{\delta x^{2}} \cdot \int_{0}^{\tau} \int_{\mathbb{R}} \frac{1}{(\tau-u)^{1 / 2}} \frac{1}{u^{3 / 4}} \exp \left[-c_{1} \frac{(x-y)^{2}}{T-u}\right] \exp \left[-c_{1} \frac{(\log (S-\varepsilon z)-y)^{2}}{u}\right] d y d u
\end{aligned}
$$

From [19] p.15, we conclude that the last expression is equal to

$$
c(\delta) \cdot e^{\delta x^{2}} \tau^{1 / 4} \exp \left[-c_{1} \frac{(x-\log (S-\varepsilon z))^{2}}{\tau}\right] .
$$


An analogous estimate holds for the second integral. Notice also that the function

$$
B(y):=\int_{-1}^{1 \wedge \frac{1}{y}} \varphi(z)(\log (1-y z))^{1 / 2} d z
$$

is continuous on $\mathbb{R}_{+}$and satisfies

$$
B(0)=0, \quad|B(x)|<c\left(1+|\log x|^{1 / 2}\right)
$$

We can now finish the estimation. For $i=2, \ldots, m$ and $\varepsilon \in(0,1]$, the following inequality holds almost surely for each $t \in[0, \bar{t}]$

$$
\begin{aligned}
& \left|\int_{0}^{\tau} \int_{\mathbb{R}} \hat{p}(\tau, x ; u, y) \hat{a}^{2}(u, y) \hat{\beta}^{i}(u, y)\left(D_{y} \hat{C}^{\varepsilon}(u, y)-D_{y} \hat{C}(u, y)\right) d y d u\right| \\
& \leq c(\delta) \cdot \lambda_{2}^{2} \cdot e^{c \cdot \lambda_{1}} \cdot e^{\delta x^{2}} \cdot B\left(\frac{\varepsilon}{S}\right) \cdot \\
& \quad\left(\sup _{z \in[-1,1]} \exp \left(-c_{1} \frac{(x-\log (S-z))^{2}}{\tau}\right)+\exp \left(-c_{1} \frac{(x-\log S)^{2}}{\tau}\right)\right)
\end{aligned}
$$

The last expression vanishes as $\varepsilon \rightarrow 0$. This implies

$$
\hat{\nu}^{\varepsilon, i}(\tau, x) \rightarrow \int_{\mathbb{R}} \int_{0}^{\tau} \hat{p}(\tau, x ; u, y) \hat{\beta}^{i}(u, y) L_{y} \hat{C}(u, y) d u d y=: \hat{\nu}^{i}(\tau, x)
$$

almost surely for any $t \in[0, \bar{t}]$. Notice that, due to the properties of function $B(y)$, the right hand side of (47) is bounded over all $S>0$ and $\varepsilon \in(0,1]$, by $c(\tau, x) \lambda_{2}^{2}(t) e^{c \cdot \lambda_{1}(t)}$. Which yields convergence in $\mathcal{H}^{2}$ sense, i.e.

$$
\mathbb{E} \int_{0}^{t}\left(\hat{\nu}_{u}^{\varepsilon, i}(\tau, x)-\hat{\nu}_{u}^{i}(\tau, x)\right)^{2} d u \rightarrow 0
$$

For $i=1$ we have to estimate the additional term

$$
\left|\hat{p}(\tau, x ; u, y) S \sigma F_{\varepsilon}^{\prime}\left(S-e^{y}\right)\right| \leq S \sigma \hat{p}(\tau, x ; u, y)
$$

Recall that $F_{\varepsilon}^{\prime} \rightarrow F_{0}^{\prime}=\mathbf{1}_{[0, \infty)}$ almost everywhere. Then, using (47), and the dominated convergence theorem, we have, as $\varepsilon \rightarrow 0$, that $\hat{\nu}^{\varepsilon, 1}(\tau, x)$ converges toward

$$
\int_{\mathbb{R}} \int_{0}^{\tau} \hat{p}(\tau, x ; u, y) \hat{\beta}^{1}(u, y) L_{y} \hat{C}(u, y) d u d y+\int_{\mathbb{R}} \hat{p}(\tau, x ; 0, y) S \sigma F_{0}^{\prime}\left(S-e^{y}\right) d y
$$

which we define as $\hat{\nu}^{1}(\tau, x)$. Since

$$
\left|\int_{\mathbb{R}} \hat{p}(\tau, x ; 0, y) S \sigma F_{0}^{\prime}\left(S-e^{y}\right) d y\right| \leq S \sigma
$$

and $\mathbb{E} \int_{0}^{t} S_{u}^{2} \sigma_{u}^{2} d u<\infty$, we can apply dominated convergence again and show that $\hat{\nu}^{\varepsilon, 1} \rightarrow \hat{\nu}^{1}$ in $\mathcal{H}^{2}$ sense. Notice that $\hat{\nu}_{t}^{i}$,s are the solutions of

$$
\left\{\begin{array}{l}
\partial_{\tau} \hat{\nu}_{t}^{i}=L_{x} \hat{\nu}_{t}^{i}+\hat{\beta}_{t}^{i} L_{x} \hat{C}_{t}, \quad \tau>0, x \in \mathbb{R}, \quad i=1, \ldots, m \\
\hat{\nu}_{t}^{1}(0, x)=S_{t} \sigma_{t} \mathbf{1}_{\left(-\infty, \log S_{t}\right]}(x) \\
\hat{\nu}_{t}^{i}(0, x)=0, \quad i=2, \ldots, m
\end{array}\right.
$$


Now, in a similar way, we pass to limit for $\hat{\mu}^{\varepsilon}$, as $\varepsilon \rightarrow 0$.

$$
\begin{aligned}
\hat{\mu}^{\varepsilon}(\tau, x)=\int_{\mathbb{R}} \int_{0}^{\tau} \hat{p}(\tau, x ; u, y) \hat{\alpha}(u, y) \hat{a}^{2}(u, y) D_{y} \hat{C}^{\varepsilon}(u, y) d u d y \\
+\int_{\mathbb{R}} \int_{0}^{\tau} \hat{p}(\tau, x ; u, y) \hat{a}^{2}(u, y)\left(\hat{\beta}(u, y) \cdot D_{y} \hat{\nu}^{\varepsilon}(u, y)\right) d u d y \\
+\frac{1}{2} S^{2} \sigma^{2} \int_{\mathbb{R}} \hat{p}(\tau, x ; 0, y) F_{\varepsilon}^{\prime \prime}\left(S-e^{y}\right) d y
\end{aligned}
$$

Consider the first term in (49). For any $\tau>0$, as $\varepsilon \rightarrow 0$, it converges to a similar expression, with $\hat{C}$ instead of $\hat{C}^{\varepsilon}$. The proof is absolutely analogous to the derivation of (47), since the only difference between the two is that $\hat{\beta}$ has been substituted for $\hat{\alpha}$. Thus, we obtain

$$
\begin{gathered}
\left|\int_{\mathbb{R}} \int_{0}^{\tau} \hat{p}(\tau, x ; u, y) \hat{\alpha}(u, y) \hat{a}^{2}(u, y)\left(D_{y} \hat{C}^{\varepsilon}(u, y)-D_{y} \hat{C}(u, y)\right) d u d y\right| \\
\leq c(\tau, x) \lambda_{2}^{2}(t) e^{c \cdot \lambda_{1}(t)}
\end{gathered}
$$

We now prove that the second term in (49) converges to

$$
\int_{\mathbb{R}} \int_{0}^{\tau} \hat{p}(\tau, x ; u, y) \hat{a}^{2}(u, y)\left(\hat{\beta}(u, y) \cdot D_{y} \hat{\nu}(u, y)\right) d u d y
$$

To give an analogous estimate in this case, we first need to find bounds on $D_{x} \hat{\nu}^{\varepsilon, i}-D_{x} \hat{\nu}^{i}$. Using (47) and the estimates on the derivatives of fundamental solution, we again follow the proof of (47) and obtain the following estimate for $i=2, \ldots, m$

$$
\begin{aligned}
& \left|D_{x} \hat{\nu}^{\varepsilon, i}(\tau, x)-D_{x} \hat{\nu}^{i}(\tau, x)\right| \\
& \quad=\left|\int_{0}^{\tau} \int_{\mathbb{R}} e^{x-y} D_{y} \hat{q}(u, y ; \tau, x) \hat{a}^{2}(u, y) \hat{\beta}^{i}(u, y)\left(D_{y} \hat{C}^{\varepsilon}(u, y)-D_{y} \hat{C}(u, y)\right) d y d u\right| \\
& =\frac{1}{2}\left|\int_{0}^{\tau} \int_{\mathbb{R}} e^{x} \partial_{y} \hat{q}(u, y ; \tau, x)\left(\partial_{y}+1\right)\left(e^{-y} \hat{a}^{2}(u, y) \hat{\beta}^{i}(u, y)\left(D_{y} \hat{C}^{\varepsilon}(u, y)-D_{y} \hat{C}(u, y)\right)\right) d y d u\right| \\
& \leq c(\delta) \cdot \lambda_{2}^{2} \cdot e^{c \cdot \lambda_{1}} \cdot e^{\delta x^{2}} \cdot \frac{1}{\tau^{3 / 4}} \int_{-1}^{1 \wedge \frac{S}{\varepsilon}} \varphi(z)\left(\log \left(1-\frac{\varepsilon}{S} z\right)\right)^{1 / 2} \cdot \\
& \quad\left(\exp \left(-c \frac{(x-\log (S-\varepsilon z))^{2}}{\tau}\right)+\exp \left(-c \frac{(x-\log S)^{2}}{\tau}\right)\right) d z
\end{aligned}
$$

For some small enough $\delta>0$. For $i=1$, the same estimate holds, with the right hand side multiplied by $1+S \sigma$. To see this, notice that the only additional term appearing in the case $i=1$ is:

$$
\frac{1}{2} S \sigma e^{x}\left|\int_{\mathbb{R}} \partial_{y} \hat{q}(0, y ; \tau, x) e^{y} F_{\varepsilon}^{\prime \prime}\left(S-e^{y}\right) d y-\partial_{y} \hat{q}(0, \log S ; \tau, x)\right|
$$

and we can proceed as for (47). Now we can prove convergence of the second term in (49), as it was done in (47). For $i=1, \ldots, m$, we have

$$
\begin{gathered}
\left|\int_{\mathbb{R}} \int_{0}^{\tau} \hat{p}(\tau, x ; u, y) \hat{a}^{2}(u, y) \hat{\beta}^{i}(u, y) \cdot\left(D_{y} \hat{\nu}^{\varepsilon, i}(u, y)-D_{y} \hat{\nu}^{i}(u, y)\right) d u d y\right| \\
\leq c(\tau, x) \cdot \lambda_{2}^{2} \cdot e^{c \cdot \lambda_{1}} \cdot(1+S \sigma)
\end{gathered}
$$


Next, we show that the last term in (49) converges toward

$$
\frac{1}{2} S \sigma^{2} \hat{p}(\tau, x ; 0, \log S) .
$$

To estimate their difference we proceed again as in (47)

$$
\begin{aligned}
\mid \frac{1}{2} S^{2} \sigma^{2} & \int_{\mathbb{R}} \hat{p}(\tau, x ; 0, y) F_{\varepsilon}^{\prime \prime}(S-\exp (y)) d y-\frac{1}{2} S \sigma^{2} \hat{p}(\tau, x ; 0, \log S) \mid \\
& \leq \frac{1}{2} S^{2} \sigma^{2} \int_{-1}^{1}\left|\frac{1}{S-\varepsilon y} \hat{p}(\tau, x ; 0, \log (S-\varepsilon y))-\frac{1}{S} \hat{p}(\tau, x ; 0, \log S)\right| \varphi(y) d y \\
& \leq \frac{1}{2} S^{2} \sigma^{2} \varepsilon \cdot \sup _{S>0}\left|\partial_{S}\left(\frac{1}{S} \hat{p}(\tau, x ; 0, \log S)\right)\right| \\
& \leq \varepsilon \cdot c(\tau, x) \cdot \sigma^{2} S^{2} \cdot e^{c \cdot \lambda_{1}}
\end{aligned}
$$

Collecting together (52), (53) and (54), we have, almost surely for every $t \in[0, \bar{\tau}]$, as $\varepsilon \rightarrow 0$

$$
\lim _{\varepsilon \rightarrow 0} \hat{\mu}_{t}^{\varepsilon}(\tau, x)=\breve{\mu}_{t}(\tau, x)+\frac{1}{2} S_{t} \sigma_{t}^{2} \hat{p}_{t}\left(\tau, x, 0, \log S_{t}\right)
$$

which we define as $\hat{\mu}_{t}(\tau, x)$, where we used

$$
\breve{\mu}(\tau, x)=\int_{\mathbb{R}} \int_{0}^{\tau} \hat{p}(\tau, x ; u, y) \hat{a}^{2}(u, y)\left(\hat{\alpha}(u, y) D_{y} \hat{C}(u, y)+\hat{\beta}(u, y) \cdot D_{y} \hat{\nu}(u, y)\right) d u d y
$$

It is clear from the previous estimates that we also have almost surely for every $t \in[0, \bar{t}]$

$$
\lim _{\varepsilon \rightarrow 0} \int_{0}^{t}\left|\hat{\mu}_{u}^{\varepsilon}(\tau, x)-\hat{\mu}_{u}(\tau, x)\right| d u=0 .
$$

Thus, since we are free to choose $\bar{t}$ and $\bar{\tau}$, for every fixed $(\tau, x)$, we have constructed predictable processes $\left\{\hat{\nu}_{t}(\tau, x), \hat{\mu}_{t}(\tau, x)\right\}_{t \geq 0}$ such that almost surely for every $t$, the random variables $\hat{\nu}_{t}$ and $\hat{\mu}_{t}$ take values in $C^{1,2}\left(\mathbb{R}_{++}, \mathbb{R}\right)$, by construction. In the process, we have also shown that, almost surely, $\hat{C}_{t}^{\varepsilon}$ and $\int_{0}^{t} \hat{\mu}_{u}^{\varepsilon} d u$ converge to $\hat{C}_{t}$ and $\int_{0}^{t} \hat{\mu}_{u} d u$ respectively. In addition, $\hat{\nu}^{\varepsilon}$ converges to $\hat{\nu}$ in $\mathcal{H}^{2}$ sense. All this implies that there exists a subsequence $\varepsilon_{n} \searrow 0$, such that $\int_{0}^{t} \hat{\nu}_{u}^{\varepsilon_{n}} \cdot d B_{u} \rightarrow \int_{0}^{t} \hat{\nu}_{u} \cdot d B_{u}$ in probability. Now, applying the same argument as in the proof of Lemma 1, we obtain for each $(\tau, x)$ the desired decomposition

$$
\hat{C}_{t}(\tau, x)=\hat{C}_{0}(\tau, x)+\int_{0}^{t} \hat{\mu}_{u}(\tau, x) d u+\int_{0}^{t} \hat{\nu}_{u}(\tau, x) \cdot d B_{u}
$$

and this completes the proof.

\section{APPENDix B}

\section{Proof of Proposition 1}

Since we consider $\tau \searrow 0$, we can assume that $\tau \leq T$ for some positive constant $T$, and as before, we will sometimes drop the subscript $t$ to streamline the notation. Recall the decomposition

$$
\hat{\mu}_{t}(\tau, x)=\bar{\mu}_{t}(\tau, x)+\frac{1}{2} S_{t} \sigma_{t}^{2} \hat{p}_{t}\left(\tau, x, 0, \log S_{t}\right)
$$


where the definition of $\bar{\mu}$ is given in (58). If we fix $\omega, t$ and $h \in C_{0}^{\infty}(\mathbb{R})$, we have

$$
\begin{aligned}
& \begin{aligned}
\left(5 \int_{\mathbb{R}}\right) h(x) \hat{\mu}(\tau, x) d x= & \int_{\mathbb{R}} \int_{\mathbb{R}} \int_{0}^{\tau} h(x) \hat{p}(\tau, x ; u, y) \hat{\alpha}(u, y) \hat{a}^{2}(u, y) e^{y} \hat{q}(0, \log S ; u, y) d u d y d x \\
& +\int_{\mathbb{R}} \int_{\mathbb{R}} \int_{0}^{\tau} h(x) \hat{p}(\tau, x ; u, y) \hat{a}^{2}(u, y)\left(\hat{\beta}(u, y) \cdot D_{y} \hat{\nu}(u, y)\right) d u d y d x \\
& +\frac{1}{2} S \sigma^{2} \int_{\mathbb{R}} h(x) \hat{p}(\tau, x ; 0, \log S) d x .
\end{aligned}
\end{aligned}
$$

Let us first prove that the first term in (59) vanishes as $\tau$ goes to zero.

$$
\begin{aligned}
& \left|\int_{\mathbb{R}} \int_{\mathbb{R}} \int_{0}^{\tau} h(x) \hat{p}(\tau, x ; u, y) \hat{\alpha}(u, y) \hat{a}^{2}(u, y) e^{y} \hat{q}(0, \log S ; u, y) d u d y d x\right| \\
& \quad \leq c \sup _{x \in \mathbb{R}}|h(x)| \cdot \\
& \quad \int_{\mathbb{R}} \int_{\mathbb{R}} \int_{0}^{\tau} \frac{1}{\sqrt{\tau-u}} \exp \left(-c \frac{(x-y)^{2}}{\tau-u}\right) \frac{1}{\sqrt{u}} \exp \left(2|y|-c \frac{(y-\log S)^{2}}{u}\right) d u d y d x \\
& \quad \leq c \cdot \sup _{x \in \mathbb{R}}|h(x)| \cdot \int_{0}^{\tau} \int_{\mathbb{R}} \frac{1}{\sqrt{u}} \exp \left(2|y|-c \frac{(y-\log S)^{2}}{u}\right) d y d u \\
& \quad \leq \tau c(S) \cdot \sup _{x \in \mathbb{R}}|h(x)| \rightarrow 0
\end{aligned}
$$

which indeed goes to 0 as $\tau \searrow 0$. As explained earlier, we denote by $c$ a generic positive constant that doesn't depend on $(\tau, x)$ and which can change every time it appears in a formula. Now we need to recall some estimates from the proof of Lemma 2.

$$
\begin{aligned}
\left|D_{x} \hat{\nu}^{i}(\tau, x)\right|= & \mid \int_{\mathbb{R}} \int_{0}^{\tau} D_{x} \hat{p}(\tau, x ; u, y) \hat{\beta}^{i}(u, y) \hat{a}^{2}(u, y) e^{y} \hat{q}(0, \log S ; u, y) d u d y \\
& +\left.\frac{1}{2} S \sigma \cdot e^{x} \cdot \partial_{z} \hat{q}(0, z ; \tau, x)\right|_{z=\log S \mid} \\
\leq & c e^{x^{2}} \int_{0}^{\tau} \int_{\mathbb{R}} \frac{1}{\tau-u} \exp \left(-c \frac{(x-y)^{2}}{\tau-u}\right) \frac{1}{u} \exp \left(-c \frac{(y-\log S)^{2}}{u}\right) d y d u \\
& +c S \sigma \cdot \frac{1}{\tau} \exp \left(-c \frac{(x-\log S)^{2}}{\tau}\right) \\
= & c\left(e^{x^{2}} \frac{1}{\sqrt{\tau}}+S \sigma \cdot \frac{1}{\tau}\right) \exp \left(-c \frac{(x-\log S)^{2}}{\tau}\right)
\end{aligned}
$$


Notice that for all small enough $\tau>0$, we have $\exp \left(-c \frac{(x-\log S)^{2}}{\tau}+x^{2}+x\right) \leq c(S)$. Using this, we estimate the second term in (59)

$$
\begin{aligned}
& \left|\int_{\mathbb{R}} \int_{\mathbb{R}} \int_{0}^{\tau} h(x) \hat{p}(\tau, x ; u, y) \hat{a}^{2}(u, y)\left(\hat{\beta}(u, y) D_{y} \hat{\nu}(u, y)\right) d u d y d x\right| \\
& \leq c(S) \cdot \sup _{x \in \mathbb{R}}|h(x)|\left[\operatorname{Leb}(\operatorname{supp}(h)) \cdot \int_{0}^{\tau} \frac{1}{\sqrt{u}} d u \int_{\mathbb{R}} \hat{p}(\tau, x ; u, y) d y\right. \\
& \left.\quad+\int_{\operatorname{supp}(h)} e^{x^{2}} \int_{\mathbb{R}} \int_{0}^{\tau} \frac{1}{u} \frac{1}{\sqrt{\tau-u}} \exp \left(-c \frac{(y-\log S)^{2}}{u}\right) \exp \left(-c \frac{(y-x)^{2}}{\tau-u}\right) d u d y d x\right] \\
& \leq \sqrt{\tau} c(S) \sup _{x \in \mathbb{R}}|h(x)|\left(\operatorname{Leb}(\operatorname{supp}(h))+\sup _{x \in \mathbb{R}}\left|e^{x^{2}}\right|\right)
\end{aligned}
$$

which goes to zero as $\tau \searrow 0$. And, due to the properties of the fundamental solution, the third term in (59) converges to $(1 / 2) S \sigma^{2} h(\log S)$ which together with (62), (62), completes the proof.

\section{Proof of Proposition 2}

For the sake of notation, we drop the subscript $t$ as it does not change throughout the proof. First fix $i=2, \ldots, m$. Then, using the results and notation introduced in these two appendices

$$
\begin{aligned}
\mid \hat{\nu}^{i}(\tau, & x)-\hat{\nu}^{i}(0, x) \mid \\
& =\left|\hat{\nu}^{i}(\tau, x)\right| \\
& \leq \int_{\mathbb{R}} \int_{0}^{\tau} e^{|y|} \hat{p}(\tau, x ; u, y) \cdot\left|\hat{\beta}^{i}(u, y) e^{-|y|}\right| \cdot\left|\hat{a}^{2}(u, y)\right| \cdot\left|D_{y} \hat{C}(u, y)\right| d u d y \\
& \leq \lambda_{2}^{2} \int_{\mathbb{R}} \int_{0}^{\tau} e^{2|y|} \hat{p}(\tau, x ; u, y) \hat{q}(0, \log S ; u, y) d u d y \\
& \leq c \lambda_{2}^{2} e^{c \cdot \lambda_{1}} e^{x^{2}} \\
& \quad \int_{\mathbb{R}} \int_{0}^{\tau} \frac{1}{(\tau-u)^{1 / 2}} \exp \left(-c \cdot \frac{(c \cdot x-y)^{2}}{T-u}\right) \frac{1}{u^{1 / 2}} \exp \left(-c \cdot \frac{(y-\log S)^{2}}{u}\right) d u d y \\
& \leq \lambda_{2}^{2} e^{c \lambda_{1}} \sqrt{\tau} e^{x^{2}} .
\end{aligned}
$$

As before, we use the notation $c$ for a positive constant which does not depend on $(t, \tau, \omega)$ and whose value can change every time it appears in a formula. For $i=1$ we need to estimate the additional term

$$
\left|\int_{\mathbb{R}} \hat{p}(\tau, x ; 0, y) S \sigma \mathbf{1}_{\left\{S-e^{y} \geq 0\right\}} d y-S \sigma \mathbf{1}_{\left\{S-e^{x} \geq 0\right\}}\right| \leq 2 S \sigma .
$$

Notice that the expression on the right side is a square integrable process. From the properties of the fundamental solution we also know that, almost surely for almost every $x \in \mathbb{R}$

$$
\lim _{\tau \searrow 0}\left|\int_{\mathbb{R}} \hat{p}(\tau, x ; 0, y) S \sigma \mathbf{1}_{\left\{S-e^{y} \geq 0\right\}} d y-S \sigma \mathbf{1}_{\left\{S-e^{x} \geq 0\right\}}\right|=0 .
$$


Now, for any $t$, using (62), we get

$$
\begin{aligned}
\mathbb{E} \int_{\mathcal{K}}\left(\int_{0}^{t}\left(\hat{\nu}_{u}(\tau, x)-\hat{\nu}_{u}(0, x)\right) \cdot d B_{u}\right)^{2} d x \\
=\sum_{i=1}^{m} \int_{\mathcal{K}} \mathbb{E} \int_{0}^{t}\left(\hat{\nu}_{u}^{i}(\tau, x)-\hat{\nu}_{u}^{i}(0, x)\right)^{2} d u d x \\
\leq c \cdot \tau \cdot\left(\int_{\mathcal{K}} e^{x^{2}} d x\right)\left(\mathbb{E} \int_{0}^{t} e^{c \cdot \lambda_{1}(u)} \lambda_{2}^{4}(u) d u\right) \\
\quad+\int_{\mathcal{K}} \mathbb{E}\left[\int_{0}^{t}\left(\int_{\mathbb{R}} S_{u}^{2} \sigma_{u}^{2} \hat{p}_{u}(\tau, x ; 0, y) \mathbf{1}_{\left(S_{u}-e^{y} \geq 0\right)} d y-S_{u}^{2} \sigma_{u}^{2} \mathbf{1}_{\left(S_{u}-e^{x} \geq 0\right)}\right)^{2} d u\right] d x .
\end{aligned}
$$

It is clear that the first term in the expression above goes to zero as $\tau \searrow 0$. The second one does so due to estimate (62) and the dominated convergence theorem. To prove the convergence of $\hat{C}$, we notice first that, almost surely for any $t$

$$
\left|\hat{C}_{t}(\tau, x)-\left(S_{t}-e^{x}\right)^{+}\right| \leq 2 S_{t},
$$

and that

$$
\lim _{\tau \searrow 0}\left|\hat{C}_{t}(\tau, x)-\left(S_{t}-e^{x}\right)^{+}\right|=0,
$$

so that the dominated convergence theorem yields

$$
\lim _{\tau \searrow 0} \mathbb{E} \int_{\mathcal{K}}\left(\hat{C}_{t}(\tau, x)-\left(S_{t}-e^{x}\right)^{+}\right)^{2} d x=0
$$

which completes the proof.

\section{REFERENCES}

1. Y. Achdou and O. Pironneau, Computational methods for option pricing, SIAM.

2. D. Blackwell, Equivalent comparisons of experiments, Annals of Mathematical Statistics 24 (1953), 265-272.

3. D. Brigo and F. Mercurio, Displaced and mixture diffusions for analytically tractable smile models, vol. Mathematical Finance - Bachelier Congress, 2000, Springer Verlag, 2001, pp. 151-174.

4. L_ Lognormal-mixture dynamics and calibration to market volatility smiles, International Journal of Theoretical and Applied Finance 5 (2002), 427-446.

5. H. Buehler, Expensive martingales, Quantitative Finance (2007), (to appear).

6. R. Carmona, Hjm: A unified approach to dynamic models for fixed income, credit and equity markets, Paris - Princeton Lecutues in Mathematical Finance, 2005 (R. Carmona et al., ed.), Lecture Notes in Mathematics, vol. 1919, Springer Verlag, 2007, pp. 3 - 45.

7. P. Carr and D. Madan, Toward a theory of volatility trading, vol. Volatility, Risk Publications, pp. $417-427$.

8. R. Cont and J. da Fonseca, Dynamics of implied volatility surfaces, quantitative Finance 2 (2002), 45-60.

9. L. Cousot, Necessary and sufficient conditions for no-static arbitrage among european calls, Tech. report, Courant Institute, New York University, Octobber 2004.

10. M.H.A. Davis and D.G. Hobson, The range of traded option prices, Tech. report, Princeton University, July 2005.

11. E. Derman and I. Kani, The volatility smile and its implied tree, Tech. report, Quantitative Research Notes, Goldman Sachs, 1994.

12. Stochastic implied trees: Arbitrage pricing with stochastic term and strike structure of volatility, International Journal of Theoretical and Applied Finance 1 (1998), 61-110. 
13. D.G.Aronson, Non-negative solutions to linear parabolic equations, Annali de la Scuola Normale Superiore di Pisa, Classe di Scienze 22(4) (1968), 607 - 694.

14. B. Dupire, Pricing with a smile, Risk 7 (1994), 32-39.

15. V. Durrleman, From implied to spot volatility, Tech. report, Stanford University, April 2005.

16. V. Durrleman and N. El Karoui, Coupling smiles, Tech. report, Stanford University, November 2006.

17. M.R. Fengler, Semiparametric modeling of implied volatility, Lecture Notes in Statistics, Springer Verlag, 2005.

18. D. Filipovic, Consistency problems for heath-jarrow-morton interest rate models, Lecture Notes in Mathematics, vol. 1760, Springer-Verlag, 2002.

19. A. Friedman, Partial differential equations of parabolic type, 1964.

20. J. Fritz, Partial differential equations, 1982.

21. J. Jacod and P. Protter, Risk neutral compatibility with option prices, Tech. report, Université de Paris VI and Cornell University, April 2006.

22. H. Kellerer, Markov-komposition und eine anwendung auf martingale, Mathematische Annalen 198 (1972), 99-122.

23. H. Kunita, Stochastic flows and stochastic differential equations, Cambridge University Press, 1990.

24. J.P. Laurent and D. Leisen, Building a consistent pricing model from observed option prices, World Scientific, 2000.

25. R. Lee, Implied volatility: Statics, dynamics, and probabilistic interpretation, International Journal of Theoretical and Applied Finance 4 (2001), 45-89.

26.

27. T. Meis and U. Marcowitz, Numerical solutions to partial differential equations, 1978.

28. P. Protter, Stochastic integration and differential equations, 2nd ed., Springer Verlag, 2004.

29. R. Hass R. Ansorge, Konvergenz von differenzenverfahren fur lineare und nichtlineare anfangswertaufgaben, 1970.

30. J. da Fonseca R. Cont and V. Durrleman, Stochastic models of implied volatility surfaces, Economic Notes 31 (2002), no. 2.

31. D. Brigo R. Torresetti and A. Pallavicini, Implied expected tranche loss surface from cdo data, Tech. report, Banca IMI, March 2007.

32. P. Schönbucher, A market model for stochastic implied volatility, Phil. Trans. of the Royal Society, Series A 357 (1999), $2071-2092$.

33. __ Portfolio losses and the term structure of loss transitition rates: a new methodology for the pricing of portfolio credit derivatives, Tech. report, ETH Z December 2005.

34. M. Schweizer and J. Wissel, Term structures of implied volatilities: Absence of arbitrage and existence results.

35. S. Sherman, On a theorem of hardy, littlewood, polya and blackwell, Proc. National Academy of Sciences USA 37 (1951), 826-831.

36. C. Stein, Notes on the comparison of experiments, Tech. report, University of Chicago, 1951. 